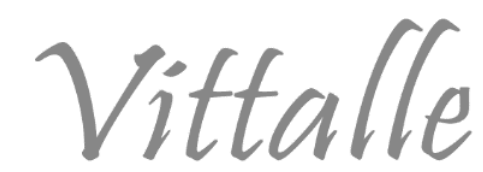

\title{
O uso de questionários na investigação do consumo mundial de bebidas alcoólicas por gestantes: uma revisão sistemática
}

\author{
Marina Veiga da Silva Amorim ${ }^{\mathrm{a}}$, Claudia Daniele Bianco ${ }^{\mathrm{b}}$, Patricia S. Brocardo ${ }^{\mathrm{a}, \mathrm{c}^{*}}$ \\ ${ }^{a}$ Programa de Pós-Graduação em Neurociências, Universidade Federal de Santa Catarina, Florianópolis, SC, Brasil \\ b Programa de Pós-Graduação em Bioquímica, Universidade Federal de Santa Catarina, Florianópolis, SC, Brasil \\ ${ }^{\mathrm{c}}$ Departamento de Ciências Morfológicas, Universidade Federal de Santa Catarina, Florianópolis, SC, Brasil
}

Histórico do Artigo
Recebido em:
14/06/2021
Aceito em:
12/11/2021
Palavras-chave:
Etanol; inquéritos e
questionários; transtornos
do espectro alcoólico
fetal; consumo de bebidas
alcoólicas; gravidez

Keywords:

Ethanol; surveys and questionnaires; fetal alcohol spectrum sisorders; alcohol drinking; pregnancy

\begin{abstract}
RESUMO
O objetivo deste artigo de revisão foi verificar quais são os instrumentos e metodologias mais utilizados para a identificação do consumo de bebidas alcoólicas por gestantes. A busca de artigos publicados entre 2010 e 2020 que investigaram o consumo de álcool em gestantes foi realizada nas bases de dados Pubmed, Medline e Web of Science de acordo com a recomendação Preferred Reporting Items for Systematic Reviews and Meta-Analyses (PRISMA). Foram localizados 1345 registros, dos quais 88 artigos foram incluídos nessa revisão. A maioria dos estudos utilizou questionários de autoria própria $(n=45)$. Entre os 16 questionários validados encontrados pode se destacar a utilização do AUDIT/AUDIT-C $(n=28)$. Baseado nessa revisão conclui-se que existem poucos estudos investigando o consumo de bebidas alcoólicas entre gestantes e a falta de padronização nos métodos de coleta dos dados torna difícil comparações entre os estudos. Sugere-se que os pesquisadores utilizem questionários validados para estudar o consumo de álcool em gestantes e que incluam medidas de intervenção nas suas pesquisas.
\end{abstract}

The use of questionnaires in the investigation of world consumption of alcoholic beverages by pregnant women: a systematic review

\begin{abstract}
The objective of this review was to verify which are the most used instruments and methodologies for the identification of alcohol consumption by pregnant women. The search for articles published between 2010 and 2020 investigated alcohol consumption in pregnant women in the Pubmed, Medline, and Web of Science databases according to the recommendation Preferred report items for systematic reviews and meta-analyses (PRISMA). A total of one thousand three hundred forty-five records were found, of which 88 manuscripts were included in this review. Most studies use questionnaires of their own $(n=45)$. Among the 16 validated questionnaires found, the use of AUDIT / AUDIT-C can be highlighted $(n=28)$. Based on this review, it is concluded that few studies are investigating the consumption of alcoholic beverages among pregnant women and the lack of standardization in the methods of data collection makes comparisons between studies difficult. Therefore, it is suggested that researchers use validated questionnaires to study alcohol consumption in pregnant women and include intervention measures in their research.
\end{abstract}

\section{Introdução}

A exposição pré-natal ao álcool (EPA) é reconhecida como a causa evitável mais comum de deficiência mental no mundo ocidental, podendo ocasionar inúmeros efeitos adversos no desenvolvimento do feto, incluindo deficiências no crescimento (comprimento e peso), malformações congênitas, distúrbios neuropsiquiátricos e alterações mentais (como deficiências neurocomportamentais e/ou de aprendizagem), que podem persistir ao longo da vida do indivíduo afetado (1).

Os efeitos da EPA são distintos dependendo do trimestre gestacional e da dose de álcool

\footnotetext{
*Autor correspondente: patricia.brocardo@ufsc.br; patibrocardo@gmail.com (Brocardo P. S.)
} 
consumida (2). As diversas alterações resultantes da EPA levaram ao estabelecimento do termo Transtornos do Espectro Alcoólico Fetal (TEAF) que compreende um conjunto de condições que podem ocorrer num indivíduo cuja mãe tenha consumido álcool durante a gravidez (3). A Síndrome Alcoólica Fetal (SAF) é a forma mais grave de TEAF e é decorrente do consumo crônico de altas doses de álcool. A SAF tem critérios diagnósticos específicos que incluem retardo no crescimento, dismorfologias faciais e alterações no sistema nervoso central (SNC) como anormalidades neurológicas, atraso no desenvolvimento e deficiências intelectuais (4).

As anormalidades neurológicas presentes entre crianças expostas ao álcool durante a gestação podem resultar em um comportamento alterado, como maior impulsividade e agressividade (5), além de a uma maior probabilidade de envolvimento com situações criminosas na vida adulta $(6,7)$. Estima-se que jovens com TEAF têm 19 vezes mais probabilidade de serem encarcerados (8). Além disso, quanto mais cedo a exposição a alguma droga, maiores as chances desse indivíduo tornar-se um dependente químico na vida adulta. Esses dados ressaltam a necessidade de estratégias de prevenção do consumo de álcool na gestação. Além disso, o diagnóstico correto para as crianças já expostas ao álcool no período perinatal é fundamental para proporcionar um tratamento adequado multidisciplinar para auxiliar no seu neurodesenvolvimento.

A prevalência mundial do uso de bebidas alcoólicas por gestantes foi estimada em $9,8 \%$ (9). No Brasil o consumo de álcool durante a gestação é estimado em $15 \%$, sendo que $3 \%$ têm um padrão de consumo do tipo beber pesado $(10,11)$. Vale ressaltar que esses dados de prevalência podem ser muito distintos entre os estudos, em função dos instrumentos utilizados para mensuração e por essa razão é importante a padronização dos questionários. Ainda, dados do ano de 2017 mostram que 17,8\% das gestantes brasileiras consumiram alguma quantidade de álcool ao longo da gravidez (10). Além disso, a prevalência global de TEAF foi estimada em 7,7 para cada 1000 indivíduos nascidos vivos, sendo que no Brasil esse número fica entre 15 e 20 para cada 1000 nascimentos (11). Desta maneira, pode-se dizer que o uso do álcool durante a gestação é uma problemática evitável, devendo ser motivo de acurada investigação durante $o$ acompanhamento pré-natal $(12,13)$.

Dentre as causas que levam à ingestão de bebidas alcoólicas durante a gestação, destacase a falta de conhecimento da gravidez, ausência de conscientização sobre o tema e de assistência adequada no período pré-natal (14). Para a detecção do consumo de bebidas alcoólicas durante a gestação pode-se utilizar questionários, sendo uma forma de avaliação sem grandes custos (15). Estes questionários, em sua maioria, são destinados a caracterizar ou avaliar a quantidade, frequência, intensidade e padrão de consumo de bebidas alcoólicas, sendo aplicados por um entrevistador devidamente treinado ou na forma de autorrelato.

O objetivo principal desta revisão foi investigar quais questionários estão sendo utilizados mundialmente para a identificação do consumo de bebidas alcoólicas por gestantes. Além de verificar e especificar quais são os questionários mais utilizados, também foram analisadas, quando relatadas nos artigos, as intervenções aplicadas ao público-alvo. A coleta e discussão destes dados é relevante para o planejamento e a realização de futuras pesquisas e intervenções, tanto no Brasil quanto em outros países, que visem prevenir o consumo de bebidas alcóolicas durante a gestação.

\section{Materiais e Métodos}

Este estudo é uma revisão integrativa da literatura sobre os instrumentos do tipo questionário utilizados na investigação do consumo mundial de bebidas alcoólicas por 
gestantes. Duas revisoras trabalharam de forma independente na condução do trabalho. O relato da presente revisão está em consonância com a recomendação Preferred Reporting Items for Systematic Reviews and Meta-Analyses (PRISMA).

Consideraram-se elegíveis estudos sobre o consumo de bebidas alcoólicas durante a gestação publicados entre 2010 e 2020. Foram considerados os artigos nos idiomas inglês e português, documentos científicos publicados na forma de artigos originais e apenas estudos que aplicaram algum tipo de questionário para rastrear a ingestão de álcool na população de mulheres gestantes.

Os estudos foram identificados nas bases de dados Pubmed Central, Medline (National Library of Medicine) e Web of Science. A busca foi realizada em outubro de 2018 e uma atualização ocorreu em março de 2020. A estratégia de busca incluiu a combinação dos seguintes termos/descritores em língua inglesa: "(Pregnancy OR pregnant OR gravid) AND (questionnaires OR instruments) AND (identify problem drinking OR alcohol consumption) AND (human) NOT (review)". Os estudos encontrados foram organizados usando o software End-Note X8® (Thomson Reuters, Filadélfia, EUA), um gerenciador de referências bibliográficas que permite a eliminação de referências duplicadas e a criação de um banco de dados para o controle da distribuição das referências selecionadas.

Os estudos identificados foram submetidos a um processo de triagem por meio da leitura dos títulos e resumos. Na sequência, os registros elegidos foram submetidos aos critérios de inclusão e exclusão, procedendo-se a leitura e a análise criteriosa do texto completo. Foram incluídos nesta revisão, (i) apenas estudos primários, (ii) artigos que realizaram alguma forma de questionário para avaliar o consumo de bebidas alcoólicas durante a gravidez, (iii) manuscritos completos disponíveis eletronicamente. Foram excluídos (i) registros em idiomas diferentes do inglês ou português, (ii) artigos de meta-análise, revisão sistemática, revisão de literatura, trabalhos de conclusão de curso, dissertações, teses e resumos de anais de congressos, (iii) pesquisas que utilizaram animais, (iv) estudos com populações restritas (ex: todos os indivíduos portadores de alguma doença), (v) trabalhos que focaram na dieta durante a gestação sem perguntas específicas sobre o consumo de bebidas alcoólicas, (vi) com data anterior a 2010.

As informações extraídas dos artigos foram: autores e ano de publicação; tamanho amostral e população analisada; questionário utilizado; local e forma de aplicação do questionário (autorrelato ou com um entrevistador); outras análises realizadas além da aplicação do questionário; informações sobre a ingestão de outras drogas além do álcool; sugestão ou realização de alguma medida de intervenção; período da gestação em que foi aplicado o questionário; principais achados da pesquisa.

Os dados inicialmente foram tabulados e distribuídos conforme os países onde as pesquisas foram realizadas. Na sequência, registrou-se quais os questionários validados cientificamente foram utilizados nos estudos. As principais informações sobre aplicação e validação destas ferramentas foram sumarizadas na forma de quadros. As informações extraídas de cada estudo foram agrupadas em dois quadros distintos segundo o questionário utilizado no estudo: questionários validados ou questionários próprios. Além disso, foram consideradas as diferentes abordagens encontradas nos estudos: outras formas de identificação do consumo de álcool além do questionário; pesquisas que incluíram dados dos recém-nascidos; estudos que incluíram pesquisa sobre consumo de bebidas alcoólicas pelos parceiros das gestantes; estudos com acompanhamento da gestante e da progênie; pesquisas realizadas fora de hospitais e clínicas.

\section{Resultados}

A estratégia da pesquisa recuperou 1345 registros, dos quais 214 estavam duplicados. Após triagem do título e resumo, selecionaram-se 204 artigos para leitura de texto 
completo. Destes, 88 estudos atenderam aos critérios de elegibilidade e foram selecionados para extração dos dados. Os detalhes do processo de seleção estão ilustrados na Figura 1.

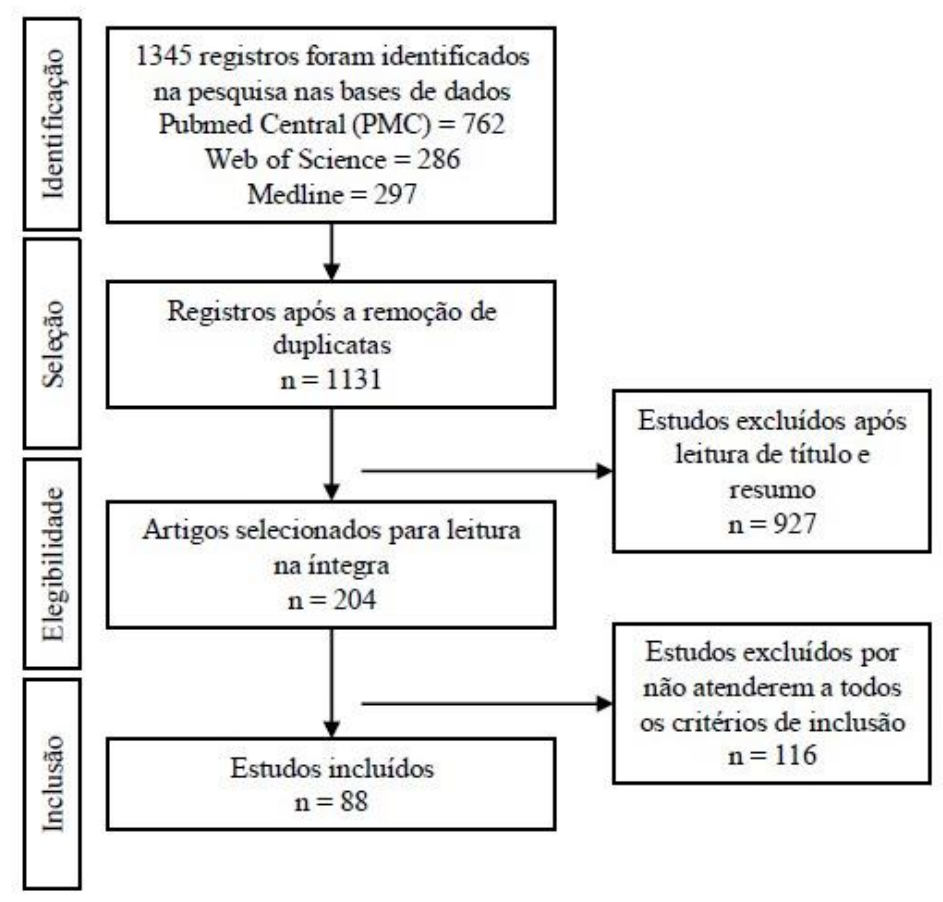

Figura 1 - Fluxograma das fases de identificação, triagem e seleção de artigos.

Foram identificados dezesseis questionários validados para rastreio do consumo de álcool, que são descritos brevemente no Quadro Suplementar 1. Os instrumentos encontrados foram: 5Ps, (5 questões sobre Parents, Partner, Past, Presen, Peerst); ASSIST (Alcohol, Smoking and Substance Involvement Screening Test); AUDIT, Alcohol Use Disorders Identification Test); AUDIT-C; CAGE (Cut-down; Annoyed; Guilty; Eyeopener); CRAFFT (Car; Relax; Alone; Forget; Friends; Trouble); NIDA (National Institute on Drug Abuse); SURP-P (Substance Use Risk Profile-Pregnancy); T-ACE (Tolerance; Annoyed; Cut-down; Eye-opener); GREEN PAGE; RAPS-QF (Rapid Alcohol Problems Screen - Quantity Frequency); Q-F (Standard quantity-frequency); SAQ (The Student Alcohol Questionnaire); TLFB (Timeline Followback); TWEAK (Tolerance; Worry; Eye-opener; Annoyed; Cut-down); WIDUS (Wayne Indirect Drug Use Screener). Quatro destes questionários estão validados na população brasileira: ASSIST (16); AUDIT (17-19); CAGE (20) e T-ACE (21). As principais características de cada questionário validado encontram-se no Quadro Suplementar 1 e as referências primárias vinculadas aos testes citados estão disponíveis no Quadro 2 do material suplementar.

Os estudos foram realizados em trinta e dois países distintos, sendo que uma pesquisa registrou dados de mais de um país (22). Analisando o número de estudos em cada país individualmente, o maior número de registros ocorreu nos Estados Unidos da América (EUA), com dezesseis ocorrências (23-38), sendo uma no Alasca (30). No entanto, avaliando a distribuição dos registros por continentes, a maior parte das pesquisas foram realizadas em países da Europa, com trinta e nove ocorrências, destacando-se a Inglaterra (22,39-43) e a Suécia (44-49), com seis estudos cada, seguida da Holanda (50-53), Irlanda (22,54-56) e Noruega (57-60), com quatro estudos cada. No Brasil foram 
registrados cinco estudos $(5,61-64)$.

Dentre os oitenta e oito estudos incluídos nesta revisão, menos da metade, quarenta e três, usaram questionários validados. Os registros mais frequentes de trabalhos usando um questionário de maneira isolada foram com o AUDIT ou AUDIT-C com dezoito ocorrências $(27,29,35,44,48,49,55,59,62,65-73)$, seguido do T-ACE com seis ocorrências $(25,37,38,60,61,74)$. Dois estudos utilizaram o teste CAGE $(26,75)$. Para os demais testes usados uma ocorrência foi registrada: ASSIST (76), GREEN PAGE (77) e TLFB (78). Além disso, quatorze estudos usaram protocolos combinados $(5,24,31,43,45-$ 47,64,79-84). A descrição detalhada de cada artigo que usou um destes questionários, individualmente ou de forma combinada, se encontra na Quadro 1.

Quadro 1 - Pesquisas que utilizaram questionários validados

\begin{tabular}{|c|c|c|c|}
\hline Questionário & Referência/País & População & $\begin{array}{l}\text { Período Gestacional } \\
\text { Avaliado } \\
\end{array}$ \\
\hline ASSIST-LITE & 76/Tailândia* & Gestantes $(\mathrm{n}=3578)$ & $\begin{array}{l}3 \text { meses antes da } \\
\text { concepção até o período } \\
\text { da entrevista }\end{array}$ \\
\hline \multirow{18}{*}{$\begin{array}{l}\text { AUDIT/ } \\
\text { AUDIT-C }\end{array}$} & 27/EUA & $\begin{array}{l}\text { Puérperas de neonatos/ } \\
\text { peso } \leq 1.500 \mathrm{~g}(\mathrm{n}=80)\end{array}$ & Período completo \\
\hline & 29/EUA & $\begin{array}{l}\text { Puérperas de neonatos } \\
\text { com peso } \leq 1.500 \mathrm{~g}(\mathrm{n}=62)\end{array}$ & Período completo \\
\hline & 35/EUA* & $\begin{array}{l}\text { Gestantes positivas para } \\
\text { alguma droga de abuso } \\
(\mathrm{n}=102)\end{array}$ & Período completo \\
\hline & 44/Suécia* & $\begin{array}{l}\text { Gestantes } 1^{\circ} . \text { trimestre } \\
(\mathrm{n}=2.264)\end{array}$ & $\begin{array}{l}\text { Até o período da } \\
\text { entrevista }\end{array}$ \\
\hline & 48/Suécia & Gestantes $(n=291)$ & $\begin{array}{l}\text { Antes da concepção e } \\
\text { período gestacional } \\
\text { completo }\end{array}$ \\
\hline & 49/Suécia* & $\begin{array}{l}\text { Gestantes e seus parceiros } \\
(\mathrm{n}=22.922)\end{array}$ & $1^{\circ}$ trimestre \\
\hline & 55/Irlanda & Gestantes $(n=300)$ & $\begin{array}{l}12 \text { meses antes da } \\
\text { concepção até o período } \\
\text { da entrevista }\end{array}$ \\
\hline & 59/Noruega* & $\begin{array}{l}\text { Gestantes e seus parceiros } \\
(\mathrm{n}=66.111)\end{array}$ & $\begin{array}{l}3 \text { meses antes da } \\
\text { concepção até a } 30^{\text {a }} \\
\text { semana de gestação }\end{array}$ \\
\hline & 62/Brasil & Puérperas $(\mathrm{n}=157)$ & Período completo \\
\hline & 65/Austrália* & Gestantes $(\mathrm{n}=1.363)$ & $\begin{array}{l}\text { Até o período da } \\
\text { entrevista }\end{array}$ \\
\hline & 66/França & $\begin{array}{l}\text { Gestantes e puérperas } \\
(\mathrm{n}=3.603)\end{array}$ & $\begin{array}{l}\text { Período gestacional } \\
\text { completo e pós-natal }\end{array}$ \\
\hline & 67/Espanha* & Gestantes $(n=451)$ & Período completo \\
\hline & 68/África do Sul* & $\begin{array}{l}\text { Gestantes com risco de } \\
\text { terem bebê com TEAF } \\
(\mathrm{n}=128)\end{array}$ & $\begin{array}{l}\text { Até o período da } \\
\text { entrevista }\end{array}$ \\
\hline & 69/Tanzânia & Gestantes $(n=365)$ & $\begin{array}{l}\text { Até o período da } \\
\text { entrevista }\end{array}$ \\
\hline & 70/Nova Zelândia* & $\begin{array}{l}\text { Mulheres } 18-35 \text { anos } \\
(\mathrm{n}=1.062)\end{array}$ & $\begin{array}{l}\text { Até o período da } \\
\text { entrevista }\end{array}$ \\
\hline & 71/Escócia* & Gestantes $(n=510)$ & $\begin{array}{l}1 \text { mês antes da concepção } \\
\text { até o período atual da } \\
\text { entrevista }\end{array}$ \\
\hline & 72/Itália & $\begin{array}{l}\text { Gestantes } 3^{\circ} \text {. trimestre } \\
(\mathrm{n}=459)\end{array}$ & Período completo \\
\hline & 73/África do Sul* & $\begin{array}{l}\text { Gestantes com risco de ter } \\
\text { um bebê com TEAF } \\
(n=67)\end{array}$ & Período completo \\
\hline$C A G E$ & 26/EUA* & Puérperas $(n=488)$ & $\begin{array}{l}\text { Período gestacional } \\
\text { completo e pós-natal }\end{array}$ \\
\hline
\end{tabular}


M. V. S. Amorim et al. / Vittalle v. 33 n. 3 (2021) 112-133

\begin{tabular}{|c|c|c|c|}
\hline & 75/Uruguai & Puérperas $(\mathrm{n}=905)$ & Período completo \\
\hline GREEN PAGE & 77/Espanha & Gestantes $(n=91)$ & Período completo \\
\hline \multirow{6}{*}{$T-A C E$} & 25/EUA* & Gestantes $(n=239)$ & $\begin{array}{l}\text { Até o período da } \\
\text { entrevista }\end{array}$ \\
\hline & 37/EUA* & Puérperas $(n=12.611)$ & Período completo \\
\hline & $36 / \mathrm{EUA}^{*}$ & $\begin{array}{l}\text { Mães afro-americanas } \\
(\mathrm{n}=75)\end{array}$ & $\begin{array}{l}2 \text { semanas antes do } 1^{\circ} \text { pré- } \\
\text { natal e período } \\
\text { gestacional completo }\end{array}$ \\
\hline & 60/Noruega* & $\begin{array}{l}\text { Gestantes e parceiros } \\
(\mathrm{n}=34.122)\end{array}$ & Período completo \\
\hline & 61/Brasil* & $\begin{array}{l}\text { Gestantes } 3^{\circ} \text { trimestre } \\
(\mathrm{n}=449)\end{array}$ & $\begin{array}{l}\text { Até o período da } \\
\text { entrevista }\end{array}$ \\
\hline & 74/República do Congo* & Gestantes (n=3099) & $\begin{array}{l}\text { Antes da concepção até } 6 \\
\text { meses pós-natal }\end{array}$ \\
\hline$T L F B$ & 78/África do Sul* & $\begin{array}{l}\begin{array}{l}\text { Crianças de } 4 \text { anos } \\
(n=500) \text { e suas mães }\end{array} \\
\end{array}$ & $\begin{array}{l}\text { Período gestacional } \\
\text { completo e pós-natal }\end{array}$ \\
\hline $\begin{array}{c}\text { AUDIT; AUDIT-C; } \\
\text { TACE- } \\
\end{array}$ & 43/Inglaterra & $\begin{array}{l}\text { Gestantes } \\
\text { no } 1^{\circ} \text { pré-natal }(\mathrm{n}=409)\end{array}$ & $\begin{array}{l}\text { Antes da concepção e/ou } \\
1^{\circ} \text { trimestre }\end{array}$ \\
\hline $\begin{array}{c}\text { AUDIT; AUDIT-C; } \\
\text { TWEAK; RAPS-QF; T- } \\
\text { ACE }\end{array}$ & 81/Argentina* & $\begin{array}{l}\text { Puérperas até } 48 \text { horas } \\
(\mathrm{n}=641)\end{array}$ & Período completo \\
\hline \multirow{8}{*}{$A U D I T-C ; Q P$} & 31/EUA* & Gestantes $(n=506)$ & $\begin{array}{l}\text { Até o período da } \\
\text { entrevista }\end{array}$ \\
\hline & 45/Suécia* & Gestantes $(n=1871)$ & Período completo \\
\hline & 46/Suécia* & Gestantes $(n=1845)$ & Período completo \\
\hline & 47/Suécia* & $\begin{array}{l}\text { Gestantes a partir da } 18^{\mathrm{a}} \\
\text { semana }(\mathrm{n}=1.594)\end{array}$ & $\begin{array}{l}\text { Antes da concepção até o } \\
\text { período atual da gestação }\end{array}$ \\
\hline & 64/Brasil* & $\begin{array}{l}\text { Gestantes adolescentes } \\
(\mathrm{n}=256)\end{array}$ & $\begin{array}{l}\text { Até o período da } \\
\text { entrevista }\end{array}$ \\
\hline & 82/Austrália* & Gestantes $(n=32)$ & $\begin{array}{l}\text { Até o período da } \\
\text { entrevista }\end{array}$ \\
\hline & 83/Canadá* & Gestantes $(n=175)$ & $\begin{array}{l}\text { Até o período da } \\
\text { entrevista }\end{array}$ \\
\hline & 84/África do Sul & $\begin{array}{l}\text { Gestantes no } 1^{\circ} \text { pré-natal } \\
(\mathrm{n}=323)\end{array}$ & Período completo \\
\hline CAGE; TWEAK; T-ACE & 79/Ucrânia* & Gestantes $(\mathrm{n}=11.909)$ & $\begin{array}{l}1 \text { mês antes da concepção } \\
\text { até o período atual da } \\
\text { entrevista }\end{array}$ \\
\hline $\begin{array}{c}\text { SURP-P; WIDUS; } \\
\text { CRAFFT; 5Ps; NIDA } \\
\text { Quick Screen } \\
\end{array}$ & 24/EUA* & Gestantes $(\mathrm{n}=1.220)$ & $\begin{array}{l}30 \text { dias anteriores à data } \\
\text { da entrevista }\end{array}$ \\
\hline$T$-ACE; AUDIT; $Q P$ & 5/Brasil* & $\begin{array}{l}\text { Gestantes no } 3^{\circ} \text { trimestre } \\
(\mathrm{n}=449) \text { e grupo de mães } \\
\text { com filhos }(\mathrm{n}=56)\end{array}$ & Período completo \\
\hline$Q-F ; S A Q$ & 80/Coréia do Sul* & $\begin{array}{l}\text { Puérperas até } 2 \text { semanas } \\
(\mathrm{n}=221)\end{array}$ & $\begin{array}{l}6 \text { meses antes da } \\
\text { concepção e período } \\
\text { gestacional completo }\end{array}$ \\
\hline
\end{tabular}

Nota: *Realizou ou sugeriu alguma intervenção. Legenda: ASSIST, Alcohol, Smoking and Substance Involvement Screening Test. AUDIT, Alcohol Use Disorders Identification Test. AUDIT-C, versão resumida do AUDIT. CAGE, Cut-down; Annoyed; Guilty; Eye-opener. CRAFFT, Car; Relax; Alone; Forget; Friends; Trouble. NIDA, National Institute on Drug Abuse. EUA, Estados Unidos da América. 5Ps, 5 questões sobre "Parents, Partner, Past, Presen, Peerst". RAPS-QF, Rapid Alcohol Problems Screen - Quantity Frequency. SAQ, Student Alcohol Questionnaire. SURP-P, Substance Use Risk ProfilePregnancy. T-ACE, Tolerance; Annoyed; Cut-down; Eye-opener. TEAF, Transtornos do Espectro Alcoólico Fetal.TLFB, Timeline Followback. TWEAK, Tolerance; Worry; Eye-opener; Annoyed; Cutdown. Q-F, standard quantity-frequency. WIDUS, Wayne Indirect Drug Use Screener. QP, questionário próprio.

Os quarenta e cinco estudos restantes formularam seus próprios questionários para rastreio do consumo de álcool, dentre os quais, apenas cinco disponibilizaram este material na íntegra (56,85-88). O Quadro 2 apresenta informações mais detalhadas sobre cada um destes quarenta e cinco artigos. 
Quadro 2 - Pesquisas que utilizaram questionários próprios

\begin{tabular}{|c|c|c|}
\hline Referência/País & População & Período Gestacional Avaliado \\
\hline 89/África do Sul & $\begin{array}{l}\text { Mães de crianças do } 1^{\circ} \text { ano do EF } \\
(\mathrm{n}=250)\end{array}$ & Completo \\
\hline 90/África do Sul* & $\begin{array}{l}\text { Parceiros de gestantes }(n=565) \text { e } \\
\text { mulheres }(n=482)\end{array}$ & Até o período da entrevista \\
\hline 91/Austrália & Mães (n=4719) & Completo \\
\hline 92/Austrália & $\begin{array}{l}\text { Puérperas com } \mathrm{RN}<400 \text { g ou }<20 \\
\text { semanas gestação }(\mathrm{n}=488)\end{array}$ & Completo \\
\hline 93/Austrália* & Gestantes no pré-natal $(n=4.570)$ & Completo e pós-natal \\
\hline $\begin{array}{l}\text { 22/Austrália, Inglaterra, Irlanda, } \\
\text { Nova Zelândia }\end{array}$ & Gestantes $(\mathrm{n}=5.628)$ & Até a 15 semana de gestação \\
\hline 63/Brasil & Gestantes $(n=1.370)$ & Completo \\
\hline 94/Canadá & Puérperas $(\mathrm{n}=5.882)$ & Completo \\
\hline 95/China & $\begin{array}{l}\text { Bebês nascidos com defeitos } \\
\text { congênitos e suas mães }(n=629)\end{array}$ & Completo \\
\hline 96/Croácia* & $\begin{array}{l}\text { Crianças do } 1^{\circ} \text { ao } 4^{\circ} \text { ano do EF } \\
(\mathrm{n}=1.110) \text { e suas mães }(\mathrm{n}=917)\end{array}$ & Completo \\
\hline 85/Dinamarca\# & Gestantes $(n=3.238)$ & Completo \\
\hline 88/Dinamarca* & Gestantes $(\mathrm{n}=6.236)$ & $\begin{array}{l}1 \text { semana antes da concepção até o } \\
\text { período da entrevista }\end{array}$ \\
\hline 97/Espanha* & Puérperas $(\mathrm{n}=51)$ & Completo \\
\hline 23/EUA & $\begin{array}{l}\text { Mães de crianças de } 23 \text { meses a } 4 \\
\text { anos }(n=725)\end{array}$ & $\begin{array}{l}\text { Antes da concepção e período } \\
\text { gestacional completo }\end{array}$ \\
\hline 28/EUA & $\begin{array}{l}\text { Puérperas de neonatos prematuros } \\
\text { com peso } \leq 1.500 \mathrm{~g}(\mathrm{n}=143)\end{array}$ & $\begin{array}{l}3 \text { meses antes da concepção e } \\
\text { período gestacional completo }\end{array}$ \\
\hline 32/EUA & $\begin{array}{l}\text { Mães de bebê com } 1 \text { ano de idade } \\
(\mathrm{n}=12.284)\end{array}$ & Até a 18 semana de gestação \\
\hline 33/EUA & $\begin{array}{l}\text { Gestantes e mães de crianças } \\
\text { menores de um ano }(n=7.905)\end{array}$ & Até o período da entrevista \\
\hline 34EUA* & $\begin{array}{l}\text { Crianças do } 1^{\circ} \text { ano do } E F(n=1.278) \\
\text { e mães }(n=123)\end{array}$ & Completo \\
\hline 36/EUA* & Puérperas afro-americanas $(\mathrm{n}=517)$ & Período completo \\
\hline 30/EUA-Alasca* & Gestantes $(n=125)$ & $\begin{array}{l}\text { Antes da concepção e período } \\
\text { gestacional completo }\end{array}$ \\
\hline 98/Gana & Gestantes $(n=397)$ & Até o período da entrevista \\
\hline 99/Gana* & Gestantes $(n=397)$ & Até o período da entrevista \\
\hline 50/Holanda & $\begin{array}{l}\text { Gestantes fumantes e alcoolistas } \\
(\mathrm{n}=3.383)\end{array}$ & Completo e pós natal \\
\hline 51/Holanda & $\begin{array}{l}\text { Mães de bebê com idade } \leq 6 \text { meses } \\
(\mathrm{n}=4.125)\end{array}$ & Completo \\
\hline 52/Holanda* & Gestantes $(\mathrm{n}=4.885)$ & Completo \\
\hline 53/Holanda* & $\begin{array}{l}\text { Gestantes }(n=158) \text { e seus parceiros } \\
(\mathrm{n}=158)\end{array}$ & $\begin{array}{l}\text { Antes da concepção até o período } \\
\text { atual da entrevista }\end{array}$ \\
\hline 39/Inglaterra* & Gestantes $(\mathrm{n}=10.851)$ & Até o período da entrevista \\
\hline 40/Inglaterra* & Gestantes $(n=1.303)$ & Completo \\
\hline 41/Inglaterra* & $\begin{array}{l}\text { Gestantes }(n=12.286) \text { e mais tarde } \\
\text { seus filhos aos } 11 \text { anos }(n=6.587)\end{array}$ & Até a 18 semana de gestação \\
\hline 42/Inglaterra* & Gestantes $(n=4.610)$ & Completo \\
\hline 56/Irlanda*\# & $\begin{array}{l}\text { Puérperas de neonatos com peso } \\
\leq 500 \mathrm{~g}(\mathrm{n}=68.925)\end{array}$ & $\begin{array}{l}\text { Antes da concepção até o período } \\
\text { atual da entrevista }\end{array}$ \\
\hline 54/Irlanda* & Puérperas $(\mathrm{n}=61.241)$ & Completo \\
\hline 100/Itália* & Gestantes $(n=513)$ & Até o período da entrevista \\
\hline 101/Japão & Gestantes $(n=94.349)$ & Completo \\
\hline 102/Japão & Puérperas de neonatos $(\mathrm{n}=1.565)$ & Completo \\
\hline 57/Noruega & Gestantes $(n=46.252)$ & $\begin{array}{l}3 \text { meses antes da concepção até o } \\
\text { período atual da entrevista }\end{array}$ \\
\hline 58/Noruega* & $\begin{array}{l}\text { Gestante }(82.362) \text { e seu parceiro } \\
(\mathrm{n}=62281)\end{array}$ & $\begin{array}{l}\text { Antes da concepção e período } \\
\text { gestacional completo }\end{array}$ \\
\hline 87/Nova Zelândia\#* & $\begin{array}{l}\text { Gestantes }(\mathrm{n}=6.822) \text { e seus filhos } \\
\text { aos } 9 \text { meses }(\mathrm{n}=6.476) \text { e aos } 2 \text { anos } \\
(\mathrm{n}=6.242)\end{array}$ & Completo \\
\hline 103/Nova Zelândia* & Puérperas $(n=723)$ & Completo \\
\hline
\end{tabular}




\begin{tabular}{|lll|}
\hline 104/Polônia & $\begin{array}{l}\text { Mães de crianças com idade até 2 } \\
\text { anos }(\mathrm{n}=104)\end{array}$ & $\begin{array}{l}\text { Antes da concepção e período } \\
\text { gestacional completo }\end{array}$ \\
\hline 105/Polônia* & Puérperas $(\mathrm{n}=8.237)$ & Completo \\
\hline 86/Romênia\#* & $\begin{array}{l}\text { Puérperas até 20 anos de idade } \\
(\mathrm{n}=74)\end{array}$ & Até o período da entrevista \\
\hline 106/Suíça\#* & Puérperas $(\mathrm{n}=207)$ & Completo \\
\hline 107/Taiwan & Puérperas indígenas $(\mathrm{n}=806)$ & Completo \\
\hline 108/Zâmbia & Gestantes $(\mathrm{n}=294)$ & Não informa \\
\hline
\end{tabular}

Nota: *Realizou ou sugeriu alguma intervenção. "Questionário disponível no artigo original.

Legenda: EF, Ensino Fundamental. EUA, Estados Unidos da América. RN, recém-nascido.

Os principais achados dos oitenta e oito estudos analisados são apresentados no Quadro 3. Foram encontrados cinquenta e oito estudos que realizaram ou sugeriram algum tipo de intervenção, sendo que vinte e um estudos consideram que a própria aplicação do questionário serve para informar sobre os riscos do consumo de bebidas alcoólicas durante a gestação $(5,25,26,35,41-43,53,58-60,65,68,71,74,76,78,88,90,93,95)$. Outros dez estudos relatam que, após aplicação do questionário, forneceram informações sobre os riscos do consumo de álcool na gravidez $(37,38,40,55,64,70,79,80,98,105)$. Além disso, um estudo auxiliou as gestantes a estabelecer metas para reduzir o consumo ou abster-se de álcool durante a gestação (73). Outros onze estudos realizaram diagnóstico de crianças com TEAF através de diferentes testes e com uma equipe multiprofissional $(5,36,38,40-42,48,73,78,87,89)$. Os demais estudos sugerem modelos para futuras intervenções novas pesquisas $(24,30,39,44,45,49,52,54,56,61,67,83,86,97,100,103,106)$.

Quadro 3 - Principais achados das pesquisas analisadas

\begin{tabular}{|c|c|}
\hline Referência & Principais achados \\
\hline 5 & Crianças expostas ao álcool durante a gestação são mais desatentas e impulsivas \\
\hline 22 & $\begin{array}{l}\text { Não associação entre consumo de álcool antes de } 15 \text { semanas de gestação e o baixo peso ao nascer, } \\
\text { pré-eclâmpsia ou nascimento prematuro espontâneo }\end{array}$ \\
\hline 23 & Beber durante a gravidez foi associado a um nível mais baixo de humor positivo \\
\hline 24 & $11,9 \%$ das gestantes tiveram resultado positivo para álcool na triagem toxicológica \\
\hline 25 & Validação do T-ACE para prever o resultado do neurodesenvolvimento infantil \\
\hline 26 & Diminuição do consumo de álcool antes do nascimento da criança até os 12 meses de idade \\
\hline 27 & Maior quantidade de metabólitos de etanol nas mães que relataram o consumo de álcool \\
\hline 28 & $34 \%$ dos recém-nascidos de baixo peso foram expostos ao álcool no $1^{\circ}$. trimestre \\
\hline 29 & $\begin{array}{l}\text { Discordância do relato (11\%) das gestantes com a deteç̧ão (30\%) dos metabólitos de etanol no } \\
\text { mecônio }\end{array}$ \\
\hline 30 & $\begin{array}{l}35 \% \text { das mulheres relataram pelo menos um episódio de consumo abusivo de álcool no início da } \\
\text { gravidez }\end{array}$ \\
\hline 31 & $31 \%$ das bebedoras atuais afirmaram que continuaram a beber durante a gravidez \\
\hline 32 & $34 \%$ das mulheres relataram ter bebido pelo menos uma vez em 32 semanas de gestação \\
\hline 33 & $15,8 \%$ relataram consumo de álcool durante a gravidez \\
\hline 34 & A prevalência de SAF foi de 2,9 a 7,5 por 1.000 indivíduos \\
\hline 35 & $\begin{array}{l}\text { Houve alta prevalência de uso de várias substâncias na gravidez. Alta prevalência de uso de tabaco no } \\
\text { grupo medicamentos }(53,8 \%) \text { e combinação de medicamentos e álcool }(66,7 \%)\end{array}$ \\
\hline 36 & $\begin{array}{l}\text { O desvio das características faciais normais tem o potencial de ser usado como uma ferramenta de } \\
\text { triagem para a exposição pré-natal a algumas substâncias ilícitas }\end{array}$ \\
\hline 37 & $8 \%$ das mães relataram consumo de álcool durante os últimos 3 meses de gravidez \\
\hline 38 & O T-ACER-3 foi mais eficaz do que T-ACE na identificação do consumo de bebidas alcoólicas \\
\hline 39 & Diminuição do número de bebedoras no $1^{\circ}(15 \%)$ e no $2^{\circ} .(21 \%)$ trimestres de gravidez \\
\hline 40 & $\begin{array}{l}\text { O relato de consumo de álcool de } \leq 2 \text { unidades/semana entre mulheres foi de } 74 \% \text { antes da gravidez e } \\
\text { de } 53 \% \text { no } 1^{\circ} \text { trimestre de gestação }\end{array}$ \\
\hline 41 & $\begin{array}{l}\text { Não associação do consumo baixo de bebidas alcoólicas durante a gravidez a problemas de saúde } \\
\text { mental ou consequências acadêmicas em crianças aos } 11 \text { anos de idade }\end{array}$ \\
\hline 42 & Efeito negativo para a saúde mental das crianças expostas ao álcool no período pré-natal \\
\hline 43 & $18 \%$ das gestantes relataram beber mensalmente e $7,8 \%$ relataram beber mais frequentemente \\
\hline 44 & $89 \%$ das mulheres consumiram álcool regularmente antes da gravidez \\
\hline 45 & Diminuição da proporção de mulheres que continuaram a beber álcool durante a gravidez \\
\hline
\end{tabular}




\begin{tabular}{|c|c|}
\hline 46 & Identificação de fatores para beber durante a gravidez \\
\hline 47 & 5,5\% das mulheres continuaram consumindo álcool após o reconhecimento da gravidez \\
\hline 48 & $\begin{array}{l}\text { O consumo de álcool baixo a moderado durante a gravidez pode influenciar negativamente o } \\
\text { desenvolvimento e o comportamento infantil }\end{array}$ \\
\hline 49 & $\begin{array}{l}\text { O uso de álcool durante a amamentação foi relatado por } 60,7 \% \text { e } 69,6 \% \text { das mulheres com } 8 \text { semanas } \\
\text { e } 12 \text { meses após o parto, respectivamente }\end{array}$ \\
\hline 50 & $8,1 \%$ consumiram álcool durante a gravidez \\
\hline 51 & Uma em cada cinco mulheres na Holanda consome álcool durante a gravidez \\
\hline 52 & Maior probabilidade de continuar bebendo durante a gravidez em mulheres com nível superior \\
\hline 53 & $\begin{array}{l}72,15 \% \text { das mulheres consumiram álcool no período da gestação, onde } 54,5 \% \text { dos seus parceiros } \\
\text { também consumiram álcool nesse período }\end{array}$ \\
\hline 54 & Fatores associados ao consumo de álcool: idade materna e uso ilícito de outras drogas \\
\hline 55 & Quase $2 / 3$ das mulheres se abstiveram totalmente de álcool durante a gravidez \\
\hline 56 & $33,6 \%$ das mulheres se abstiveram do consumo de álcool antes da gravidez \\
\hline 57 & $91 \%$ consumiram álcool antes da gravidez e menos de $20 \%$ durante a gravidez \\
\hline 58 & Comparou a abstinência de álcool na gravidez entre pais primíparos e experientes \\
\hline 59 & Relatos de abstinência (72\% a 89\%) de bebida alcoólica na gestação dentre as entrevistadas. \\
\hline 60 & $\begin{array}{l}8 \% \text { das mães não fizeram uso de álcool no } 1^{\circ} \text { trimestre gestacional, enquanto } 70 \% \text { se abstiveram } \\
\text { durante os } 3 \text { meses anteriores à gestação }\end{array}$ \\
\hline 61 & $9,1 \%$ de gestantes foram identificadas com transtornos relacionados ao álcool \\
\hline 62 & A ingestão alcoólica excessiva influencia a ocorrência de nascimento prematuro \\
\hline 63 & $23 \%$ das mulheres consumiram álcool durante a gravidez \\
\hline 64 & Prevalência de $32,4 \%$ para o uso de álcool na gestação em adolescentes \\
\hline 65 & $63,2 \%$ das gestantes relataram o aconselhamento sobre consumo de álcool na gravidez \\
\hline 66 & $0,1 \%$ e $0,2 \%$ das gestantes relataram o uso diário e no último mês de álcool, respectivamente \\
\hline 67 & $\begin{array}{l}\text { Consumo de álcool nos três trimestres de gestação de } 40,7 \%, 25,5 \% \text { e } 17,1 \% \text {, respectivamente. Um } \\
\text { nível educacional mais alto foi associado a maior exposição ao álcool durante a gestação }\end{array}$ \\
\hline 68 & O conhecimento sobre TEAF diminuiu o consumo de álcool durante a gestação \\
\hline 69 & Uma prevalência de $15,1 \%$ de consumo de álcool durante a gravidez foi encontrada \\
\hline 70 & Fatores que motivam o consumo de álcool entre mulheres em idade reprodutiva \\
\hline 71 & $\begin{array}{l}\text { Análise dos biomarcadores em amostras de cabelo de gestantes conseguiu identificar consumo } \\
\text { excessivo de álcool que não havia sido relatado no teste AUDIT }\end{array}$ \\
\hline 72 & Validação da versão italiana do AUDIT-C como questionário de autorrelato \\
\hline 73 & Diminuição de 16,9 doses para 8,6 doses no $2^{\circ}$ trimestre e 8,1 doses no $3^{\circ}$ trimestre da gravidez \\
\hline 74 & $\begin{array}{l}23,3 \% \text { das mulheres atendidas no pré-natal fizeram o uso de álcool durante a gravidez e a maioria } \\
\text { (83\%) ontinuou a consumir álcool após o reconhecimento da gravidez }\end{array}$ \\
\hline 75 & 44\% de exposição pré-natal ao álcool, identificado por amostras de mecônio \\
\hline 76 & Relato de consumo de álcool de 1,2\% foi diferente dos dados bioquímicos de 7,7\% \\
\hline 77 & Cerca de $70 \%$ das mães consumiram álcool no início da gravidez \\
\hline 78 & $\begin{array}{l}\text { Associação de bebedeira na gravidez com maior risco de transtornos cognitivos e problemas } \\
\text { comportamentais em crianças aos } 4 \text { anos de idade }\end{array}$ \\
\hline 79 & $92,7 \%$ das mulheres relataram o consumo de álcool \\
\hline 80 & O álcool foi consumido durante a gravidez por $12,7 \%$ das participantes \\
\hline 81 & Comparação do consumo de álcool na gestação usando diferentes questionários \\
\hline 82 & $\begin{array}{l}\text { Triagem de álcool na gravidez gerou relato de casos de desconforto o que sugere que esse tipo de } \\
\text { abordagem no pré-natal continua problemática }\end{array}$ \\
\hline 83 & Comparou os questionários T-ACE e o TWEAK \\
\hline 84 & Foi observado uma associação significativa entre depressão, uso de substâncias e abuso de álcool \\
\hline 85 & $\begin{array}{l}\text { Foram identificados fatores de risco independentes para o consumo excessivo de álcool no início da } \\
\text { gravidez: gravidez não planejada, tabagismo e hábitos de álcool antes da gravidez }\end{array}$ \\
\hline 86 & $17,5 \%$ das gestantes consumiram álcool \\
\hline 87 & $\begin{array}{l}\text { O consumo de álcool durante a gravidez tem um efeito negativo, especialmente no temperamento } \\
\text { infantil, mesmo que pequenas quantidades de álcool sejam consumidas }\end{array}$ \\
\hline 88 & $49,4 \%$ das gestantes beberam álcool no $1^{\circ}$ mês, $6,6 \%$ no $6^{\circ}$ mês; $7,6 \%$ no $9^{\circ}$ mês de gravidez \\
\hline 89 & Análise de regressão demonstrou que beber aumenta a probabilidade de TEAF \\
\hline 90 & $54 \%$ das gestantes relataram beber álcool de $2-4$ x/mês \\
\hline 91 & $\begin{array}{l}\text { Crianças expostas ao álcool no } 1^{\circ} \text { trimestre tinham } 2 x \text { mais chances de não atingir o valor de referência } \\
\text { para leitura e escrita }\end{array}$ \\
\hline 92 & Estudo de associações de internações por consumo de álcool durante a gravidez \\
\hline 93 & $\begin{array}{l}\text { Mulheres cujos parceiros relataram dependência de álcool tiveram chances quase } 9 \text { vezes maiores de } \\
\text { sofrerem violência pelo parceiro }\end{array}$ \\
\hline 94 & $\begin{array}{l}\text { Grávidas que relataram indiferença ou estar infeliz em relação à gravidez exibiam um risco aumentado } \\
\text { de } 1,89 \text { e } 2,5 \text { vezes de beber álcool durante a gravidez, respectivamente }\end{array}$ \\
\hline
\end{tabular}




\begin{tabular}{|c|c|}
\hline 95 & $\begin{array}{l}\text { Aumento da prevalência de defeitos congênitos nos filhos de mães que consumiram álcool durante a } \\
\text { gravidez }\end{array}$ \\
\hline 96 & A exposição ao álcool durante a gravidez foi admitida por $11,5 \%$ das mães \\
\hline 97 & $\begin{array}{l}\text { 6\% das mulheres relataram o consumo de álcool durante a gravidez. Contudo, através da detecção dos } \\
\text { metabólitos de etanol no mecônio chegou a ser } 16,7 \%\end{array}$ \\
\hline 98 & 20,4\% das gestantes consumiram álcool \\
\hline 99 & $64,2 \%$ das gestantes tiveram acesso a bebida alcoólica em bares \\
\hline 100 & $74,8 \%$ da população feminina que bebia álcool antes da gravidez parou durante a gravidez \\
\hline 101 & $\begin{array}{l}\text { O consumo de } 1,0 \mathrm{~g} \text { ou mais de álcool/dia durante a gravidez foi associado a um risco aumentado de } \\
\text { parto prematuro }\end{array}$ \\
\hline 102 & Consumo pesado de álcool nos $2^{\circ}$ e $3^{\circ}$ trimestres foi associado a um risco $4 \mathrm{x}$ maior de prematuridade \\
\hline 103 & $34 \%$ das mulheres relataram beber em algum momento durante a gravidez \\
\hline 104 & $22 \%$ das participantes eram fumantes, $13 \%$ bebiam apenas álcool, e $7 \%$ fumavam e bebiam álcool \\
\hline 105 & $\begin{array}{l}55 \% \text { dos médicos que atenderam as gestantes não discutiram o consumo do álcool durante a gestação } \\
\text { e mais de } 2 \% \text { dos médicos recomendaram o consumo de álcool na gravidez }\end{array}$ \\
\hline 106 & $36,3 \%$ beberam pelo menos um copo de álcool durante a gravidez \\
\hline 107 & $26,6 \%$ das gestantes indígenas ingeriram álcool durante a gravidez \\
\hline 108 & Foram encontradas crenças relacionadas a gravidez, ao parto e ao consumo de álcool \\
\hline
\end{tabular}

Legenda: T-ACE: Tolerance; Annoyed; Cut-down; Eye-opener. TWEAK: Tolerance; Worry; Eye-opener; Annoyed; Cut-down. AUDIT, Alcohol Use Disorders Identification Test). Betel, bebida com efeitos psicoativos.

\section{Discussão}

Esta revisão reuniu oitenta e oito artigos publicados mundialmente na última década sobre o consumo de bebidas alcoólicas entre gestantes. Embora esse número seja pequeno, os resultados mostraram um crescimento de publicações nesta área. Com uma média de 8,8 artigos/ano, sendo 2013 o ano com maior número de publicações (15 artigos). Essa escassa quantidade pode ser decorrente da subestimação dos prejuízos do álcool durante o desenvolvimento, falta de investimento nessa área e de inclusão, nesta revisão, de estudos clínicos em andamento. Ainda assim foi possível observar uma ampla diversidade de publicações entre os países e uma grande variedade de metodologias.

Um dos achados desta revisão foi a falta de padronização na coleta dos dados, o que dificulta a comparação entre estudos, bem como a discussão dos dados mundialmente. A diversidade metodológica, em parte, deve-se a diferentes questionários utilizados nos estudos encontrados, sem uma padronização do teste aplicado, do período avaliado e da forma de aplicação. Ainda, o uso de questionários não validados, e que em sua maioria não foram descritos nos artigos, pode ser considerado um ponto crucial que precisa ser discutido, porque impede a replicabilidade e dificulta a discussão dos resultados coletados.

Alguns estudos relataram a associação do uso de bebidas alcoólicas com outras variáveis: presença de eventos estressantes $(38,97)$; depressão $(32,46)$; tabagismo $(26,43,51,55,57,63,94,101,104,107)$; abandono do cônjuge ou parceiro (53); crenças (108); violência doméstica $(5,63,77,107)$ e baixo apoio social $(42)$. Ainda, o nível de escolaridade baixo foi associado ao maior consumo de bebidas alcoólicas por gestantes $(62,104)$. No entanto, uma maior prevalência de consumo de álcool no final da gravidez $(66,76)$ e maior probabilidade de continuar bebendo durante a gestação $(23,51,54,79)$ foram associadas a maior escolaridade.

O consumo de bebidas alcoólicas muitas vezes está associado com o tabagismo e uso de outras drogas (109), por isso no momento da coleta dos dados sobre os hábitos da gestante, cinquenta e seis estudos $(63,6 \%)$ usados nessa revisão investigaram esse fator. No entanto $36,4 \%$ não abordaram o rastreio de outras drogas além do álcool. A importância de investigar outras drogas, deve-se a associação do consumo entre elas. $\mathrm{O}$ consumo de bebidas alcoólicas tem grande associação com o de tabaco, e muitas vezes seu consumo ocorre de forma concomitante (109). Assim, os questionários e outros meios 
de rastreio devem considerar estas questões e fazer o possível para abordar também o consumo de outras drogas além do álcool.

As causas que motivam o consumo de álcool antes do conhecimento da gravidez podem ser semelhantes aos motivos que levam mulheres não grávidas em idade reprodutiva consumirem bebidas alcoólicas. Sendo estes, pressão social, fácil disponibilidade, a vulnerabilidade ambiental e a violência doméstica (110). Ainda, o consumo de álcool durante a gravidez parece estar associado a três grupos de questões interligadas: questões subjetivas e individuais (como expectativas, motivações e dificuldade das mulheres em identificar seu próprio consumo como arriscado); questões socioculturais e ambientais (como valor cultural do álcool, fácil acesso e influência de parentes, amigos e parceiros); desinformação, opiniões inconsistentes veiculadas na mídia e falta de abordagem técnica do tema no pré-natal (111). Compreender os fatores que contribuem para o uso de álcool pelas mulheres durante a gravidez é fundamental para apoiar a saúde e o bem-estar das mulheres e prevenir o TEAF. Uma recente revisão sistemática de estudos qualitativos envolvendo mulheres grávidas e puérperas foi realizada para compreender as barreiras e facilitadores que influenciam o uso de álcool na gravidez (112). Nessa revisão os autores identificaram cinco temas que impactam o uso, abstenção e redução de álcool pelas mulheres: (1) relações sociais e normas; (2) estigma; (3) trauma e outros fatores de estresse; (4) informações e mensagens sobre álcool; e (5) acesso a cuidados e recursos essenciais confiáveis e equitativos (112). Portanto, os esforços de saúde pública que abordam o risco de beber, especialmente em contextos sociais, e o tabagismo entre mulheres em idade fértil podem diminuir a prevalência do consumo de bebidas alcoólicas por mulheres antes do conhecimento da gestação. Desta forma, evitando a exposição ao álcool durante a gestação (44). Além disso, a falta de uma boa assistência pré-natal também agrava essa situação. Em um estudo realizado na África do Sul, 30\% das gestantes relataram que não tiveram aconselhamento sobre as consequências do uso de álcool neste período (76). Já em um estudo na Suécia, 55\% dos médicos que atenderam as gestantes não discutiram as consequências do consumo do álcool durante a gestação e mais de $2 \%$ dos médicos fizeram a recomendação do consumo de álcool na gravidez (48). Ainda, outra pesquisa realizada no continente africano mostrou que 33,5\% das gestantes ouviram falar dos efeitos prejudiciais do álcool nas clínicas pré-natais (98).

É importante destacar que pesquisas realizadas fora de um contexto hospitalar (telefone, correio, online, escolas e em locais de comercialização de bebidas alcoólicas) proporcionam um alcance maior de público-alvo $(33,41,50,52,55,58,65,66,70,83,88,90,91,96,101)$. Esse tipo de coleta também aumenta a possibilidade de abrangência de como o consumo de álcool por gestantes pode e deve ser investigado, coletando dados e aumentando a intervenção sobre a problemática em questão.

A forma de intervir para diminuir o consumo de bebidas alcoólicas durante a gestação também pode ser uma etapa facilmente incluída nas pesquisas que investigam esse tema, como uma extensão destas. Foram encontradas nesta revisão várias maneiras diferentes de alertar sobre o tema logo após a aplicação do questionário $(37,38,40,55,64,70,79,80,98,99,105)$, podendo se estender aos riscos de consumir bebidas alcoólicas durante a amamentação (93). É importante também a realização de um acompanhamento mais regular e de longo prazo junto às gestantes que apresentam maior risco de serem consumidoras abusivas, e dar orientação após a realização do diagnóstico das crianças com TEAF, principalmente fora do contexto hospitalar $(73,89,95)$.

Como demonstrado anteriormente, trinta e três trabalhos (37,5\%) não apresentaram nenhuma forma de intervenção com a população avaliada. Este dado é preocupante, levando em consideração que todos os estudos incluídos nesta revisão realizaram a 
aplicação de pelo menos um questionário para rastreio de bebidas alcoólicas. Assim, deve-se refletir sobre o papel e o objetivo principal dos estudos nessa área do conhecimento, o de melhoria do bem-estar social e maior qualidade de vida para a população diretamente afetada. Portanto, as intervenções devem ser aliadas aos estudos como parte indispensável e constarem na descrição da metodologia sempre que possível.

Vale destacar que apenas cinco estudos brasileiros foram incluídos nesta revisão, três realizados no estado de São Paulo $(5,61,63)$, um no Piauí (64) e um em Santa Catarina (62). Dentre as possíveis explicações para esse número reduzido de estudos rastreados no Brasil, estima-se que três fatores podem estar envolvidos: 1) a área de pesquisa ainda é pouco estudada; 2) as pesquisas existentes encontram-se no formato de trabalhos acadêmicos não publicados (como dissertações e teses), portanto não fazem parte dos critérios de inclusão desta revisão; 3) as bases de dados escolhidas e 4) os descritores utilizados na revisão.

Os estudos brasileiros foram realizados dentro de hospitais, e tiveram em comum o uso de questionários validados (AUDIT e T-ACE) $(5,61,62,64)$, com exceção de uma pesquisa que utilizou apenas questionário próprio (63). A prevalência de consumo de bebidas alcoólicas entre gestantes e puérperas foi elevada nesses estudos: 23\% (63), $32,4 \%$ (64), 35,7\% (62). Porém, o Brasil carece de um estudo nacional multicêntrico que possa caracterizar de forma mais precisa os dados epidemiológicos relacionados ao consumo de álcool durante a gestação e dados de indivíduos diagnosticados com TEAF.

Embora tenham sido encontrados oitenta e oito estudos nessa revisão, a diversidade metodológica e as diferentes formas de apresentação dos resultados impossibilitaram a utilização de técnicas quantitativas para avaliar a prevalência e fatores associados ao consumo de álcool. Apesar disso, é possível destacar alguns dos fatores sociais de risco encontrados nessa revisão: a baixa escolaridade, violência doméstica, baixo nível socioeconômico, gravidez indesejada e a associação do consumo de outras drogas como o tabaco.

\section{Considerações Finais}

Existe uma carência de estudos padronizados para estudar o consumo de bebidas alcoólicas por gestantes. Assim, é essencial a utilização de questionários validados, pois eles agregam confiabilidade e reprodutibilidade aos estudos epidemiológicos. É esperado que o presente estudo sirva, principalmente, para refletir sobre as diversas metodologias e abordagens existentes atualmente, e como ferramenta de consulta com o intuito de padronizar as futuras pesquisas sobre o consumo de bebidas alcoólicas durante a gestação. Vários pontos abordados nesta revisão são importantes para um melhor delineamento de futuras pesquisas. destaca-se a importância da utilização de questionários validados do consumo de álcool por gestantes para posterior comparação entre os diferentes estudos. Além disso, é necessário que ocorra um incentivo para a investigação de estudos nessa área em questão. E a conscientização de realizar intervenções como uma etapa nas pesquisas deve ser parte indispensável dos estudos que avaliam o consumo de bebidas alcoólicas durante a gestação, pois podem prevenir inúmeros problemas para o indivíduo afetado, a família e a sociedade.

\section{Referências}

1. Popova S, Lange S, Burd L, Chudley AE, Clarren SK, Rehm J. Cost of Fetal Alcohol Spectrum Disorder Diagnosis in Canada. PLoS One. 2013; 8(4): e60434.

2. Mesquita $\mathrm{M}$ dos A, Segre CA de M. Congenital malformations in newborns of alcoholic mothers. Einstein (São Paulo). 2010; 8(4): 461-6 
3. Hoyme HE, May PA, Kalberg WO, Kodituwakku P, Gossage JP, Trujillo PM, et al. A practical clinical approach to diagnosis of fetal alcohol spectrum disorders: Clarification of the 1996 institute of medicine criteria. Pediatrics. 2005; 115(1): 39-47.

4. Jones KL, Smith DW. Recognition of the fetal alcohol syndrome in early infancy. Lancet. 1973; 302(7836): 999-1001.

5. Furtado EF, Roriz ST de S. Inattention and impulsivity associated with prenatal alcohol exposure in a prospective cohort study with 11-years-old Brazilian children. Eur Child Adolesc Psychiatry. 2016; 25(12): 1327-1335.

6. Streissguth AP, Barr HM, Bookstein FL, Sampson PD, Olson HC. The long-term neurocognitive consequences of prenatal alcohol exposure: A 14-Year Study. Psychol Sci. 1999; 10(3): 186-190.

7. Fast DK, Conry J. Fetal alcohol spectrum disorders and the criminal justice system. Vol. 15, Ment. Retard. Dev. Disabil. Res. Rev. 2009. 12: 70-82.

8. Popova S, Lange S, Bekmuradov D, Mihic A, Rehm J. Fetal Alcohol Spectrum Disorder prevalence estimates in correctional systems: A systematic literature review. Can J Public Heal. 2011;102(5): 33640.

9. Popova S, Lange S, Probst C, Gmel G, Rehm J. Estimation of national, regional, and global prevalence of alcohol use during pregnancy and fetal alcohol syndrome: a systematic review and meta-analysis. Lancet Glob Heal. 2017;5(3): e290-e299.

10. Lange S, Probst C, Rehm J, Popova S. Prevalence of binge drinking during pregnancy by country and World Health Organization region: Systematic review and meta-analysis. Reprod Toxicol. 2017;73: 214-21.

11. Lange S, Probst C, Gmel G, Rehm J, Burd L, Popova S. Global prevalence of fetal alcohol spectrum disorder among children and youth: A systematic review and meta-analysis. JAMA Pediatr. 2017; 171(10): 948-56.

12. Moraes CL, Reichenheim ME. Rastreamento de uso de álcool por gestantes de serviços públicos de saúde do Rio de Janeiro. Rev Saude Publica. 2007; 41(5): 695-703.

13. Maria FN, Jornada LK, Sakae TM, Cassol-Jr OJ, Sakae DY, Quevedo JL. Uso de álcool e tabaco por gestantes em maternidade do sul de Santa Catarina. Arq Catarin Med. 2015; 44(1): 41-61.

14. Tough SC, Clarke M, Clarren S. Preventing fetal alcohol spectrum disorders. Preconception counseling and diagnosis help. Can Fam Physician. 2005; 51(9): 1199-201.

15. Chang G. Alcohol-screening instruments for pregnant women. Alcohol Res Heal. 2001; 25(3): 204-9.

16. Henrique IFS, De Micheli D, Lacerda RB de, Lacerda LA de, Formigoni MLO de S. Validação da versão brasileira do teste de triagem do envolvimento com álcool, cigarro e outras substâncias (ASSIST). Rev Assoc Med Bras. 2004; 50(2): 199-206.

17. Lima CT, Freire ACC, Silva APB, Teixeira RM, Farrell M, Prince M. Concurrent and construct validity of the audit in an urban Brazillian sample. Alcohol Alcohol. 2005; 40(6): 584-9.

18. Mattara FP, Ângelo PM, Faria JB, Juliana Alvares Duarte Bonini C. Confiabilidade do teste de identificação de transtornos devido ao uso de álcool (AUDIT) em adolescentes. SMAD Rev Eletrônica Saúde Ment Álcool e Drog. 2010; 6(2): 296-314.

19. Santos WS dos, Fernandes DP, Grangeiro AS de M, Lopes GS, Sousa EMP. Medindo consumo de álcool: análise fatorial confirmatória do Alcohol Use Disorder Identification Test (AUDIT). Psico-USF. 2013; 18(1): 121-130.

20. Masur J, Monteiro MG. Validation of the "CAGE" alcoholism screening test in a Brazilian psychiatric inpatient hospital setting. Brazilian J Med Biol Res. 1983; 16(3): 215-8.

21. Fabbri CE, Furtado EF, Laprega MR. Consumo de álcool na gestação: desempenho da versão brasileira do questionário T-ACE. Rev Saude Publica. 2007;41(6): 979-84.

22. McCarthy FP, O'keeffe LM, Khashan AS, North RA, Poston L, McCowan LME, et al. Association between maternal alcohol consumption in early pregnancy and pregnancy outcomes. Obstet Gynecol. 2013; 122(4): 830-7.

23. Chen JH. Maternal alcohol use during pregnancy, birth weight and early behavioral outcomes. Alcohol Alcohol. 2012;47(6): 649-56.

24. Chang G, Ondersma SJ, Blake-Lamb T, Gilstad-Hayden K, Orav EJ, Yonkers KA. Identification of substance use disorders among pregnant women: A comparison of screeners. Drug Alcohol Depend. 2019; 205: 107651

25. Chiodo LM, Delaney-Black V, Sokol RJ, Janisse J, Pardo Y, Hannigan JH. Increased cut-point of the TACER-3 screen reduces false positives without losing sensitivity in predicting risk alcohol drinking in pregnancy. Alcohol Clin Exp Res. 2014; 38(5): 1401-8.

26. Delker K, Fisher A, Delker BC, Kim HK, Fisher PA. First Time's a Charm: Maternal Problem Drinking Around the Birth of a Child in Primiparous and Multiparous Women at Risk for Child Maltreatment. J Stud Alcohol Drugs. 2014; 75(6): 973-8. 
27. Gauthier TW, Mohan SS, Gross TS, Harris FL, Guidot DM, Brown LAS. Placental fatty acid ethyl esters are elevated with maternal alcohol use in pregnancies complicated by prematurity. PLoS One. 2015; 10(5): e0136366.

28. Gauthier TW, Guidot DM, Kelleman MS, McCracken CE, Brown LAS. Maternal Alcohol Use During Pregnancy and Associated Morbidities in Very Low Birth Weight Newborns. Am J Med Sci. 2016; 352(4): 368-75.

29. Gross TS, Harris F, Brown LAS, Gauthier TW. Ethyl linolenate is elevated in meconium of very-lowbirth-weight neonates exposed to alcohol in utero. Pediatr Res. 2017; 81(3): 461-7.

30. Khan BA, Robinson RF, Smith JJ, Dillard DA. Prenatal alcohol exposure among Alaska Native/American Indian infants. Int J Circumpolar Health. 2013;72.

31. Kiely M, Thornberry JS, Bhaskar B, Rodan MF. Patterns of alcohol consumption among pregnant African-American women in Washington, DC, USA. Paediatr Perinat Epidemiol. 2011; 25(4): 328-39.

32. Leis JA, Heron J, Stuart EA, Mendelson T. Associations between depressive and anxious symptoms and prenatal alcohol use. Matern Child Health J. 2012; 16(6): 1304-11.

33. Mårdby AC, Lupattelli A, Hensing G, Nordeng H. Consumption of alcohol during pregnancy-A multinational European study. Women and Birth. 2017; 30(4): 207-13.

34. May PA, Keaster C, Bozeman R, Goodover J, Blankenship J, Kalberg WO, et al. Prevalence and characteristics of fetal alcohol syndrome and partial fetal alcohol syndrome in a Rocky Mountain Region City. Drug Alcohol Depend. 2015; 155: 118-27.

35. Shrestha S, Jimenez E, Garrison L, Pribis P, Raisch DW, Stephen JM, et al. Dietary Intake Among Opioid- and Alcohol-Using Pregnant Women. Subst Use Misuse. 2018; 53(2): 260-9.

36. Washington KN, Bamji ZD, Yue Q, Reeves IV. Morphometric analysis in ethnic neonates from multiple substance exposure. Frontiers In Bioscience. 2019; 24: 527-544.

37. Cheng D, Kettinger L, Uduhiri K, Hurt L. Alcohol consumption during pregnancy: Prevalence and provider assessment. Obstet Gynecol. 2011; 117(2): 212-7.

38. Chiodo LM, Sokol RJ, Delaney-Black V, Janisse J, Hannigan JH. Validity of the T-ACE in pregnancy in predicting child outcome and risk drinking. Alcohol. 2010; 44(7-8): 595-603.

39. Cooper DL, Petherick ES, Wright J. The association between binge drinking and birth outcomes: results from the Born in Bradford cohort study. J Epidemiol Community Heal. 2013; 67(10): 821-8.

40. Nykjaer C, Alwan NA, Greenwood DC, Simpson NAB, Hay AWM, White KLM, et al. Maternal alcohol intake prior to and during pregnancy and risk of adverse birth outcomes: Evidence from a british cohort. J Epidemiol Community Health. 2014; 68(6): 542-9.

41. Sayal K, Draper ES, Fraser R, Barrow M, Smith GD, Gray R. Light drinking in pregnancy and midchildhood mental health and learning outcomes. Arch Dis Child. 2013; 98(2): 107-11.

42. Sayal K, Heron J, Draper E, Alati R, Lewis SJ, Fraser R, et al. Prenatal exposure to binge pattern of alcohol consumption: mental health and learning outcomes at age 11. Eur Child Adolesc Psychiatry. 2014; 23(10): 891-9.

43. Smith L, Savory J, Couves J, Burns E. Alcohol consumption during pregnancy: Cross-sectional survey. Midwifery. 2014; 30(12): 1173-8.

44. Comasco E, Hallberg G, Helander A, Oreland L, Sundelin-Wahlsten V. Alcohol Consumption Among Pregnant Women in a Swedish Sample and Its Effects on the Newborn Outcomes. Alcohol Clin Exp Res. 2012; 36(10): 1779-86.

45. Nilsen P, Holmqvist M, Bendtsen P, Hultgren E, Cedergren M. Is Questionnaire-Based Alcohol Counseling More Effective for Pregnant Women Than Standard Maternity Care? J Womens Health. 2010; 19(1): 161-7.

46. Nilsen P, Skagerström J, Rahmqvist M, Hultgren E, Blomberg M. Alcohol prevention in Swedish antenatal care: Effectiveness and perceptions of the Risk Drinking project counseling model. Acta Obstet Gynecol Scand.2012; 91(6): 736-43.

47. Skagerström J, Alehagen S, Häggström-Nordin E, Årestedt K, Nilsen P. Prevalence of alcohol use before and during pregnancy and predictors of drinking during pregnancy: A cross sectional study in Sweden. BMC Public Health. 2013;13: 780.

48. Sundelin-Wahlsten V, Hallberg G, Helander A. Higher alcohol consumption in early pregnancy or lowto-moderate drinking during pregnancy may affect children's behaviour and development at one year and six months. Acta Paediatr Int J Paediatr. 2017; 106(3): 446-53.

49. Wilson IM, Eurenius E, Lindkvist M, Edin K, Edvardsson K. Is there an association between pregnant women's experience of violence and their partner's drinking? A Swedish population-based study. Midwifery. 2019; 69:84-91.

50. Beijers C, Ormel J, Meijer JL, Verbeek T, Bockting CLH, Burger H. Stressful events and continued smoking and continued alcohol consumption during mid-pregnancy. PLoS One. 2014; 9(1): e86359.

51. Lanting CI, Van Dommelen P, Van Der Pal-De Bruin KM, Bennebroek Gravenhorst J, Van Wouwe JP. 
Prevalence and pattern of alcohol consumption during pregnancy in the Netherlands. BMC Public Health. 2015; 15: 723.

52. Pfinder M, Kunst AE, Feldmann R, Van Eijsden M, Vrijkotte TGM. Educational Differences in Continuing or Restarting Drinking in Early and Late Pregnancy: Role of Psychological and Physical Problems alcohol intake during pregnancy causes. Vol. 75, Stud. Alcohol Drugs. 2014; 75(1):47-55.

53. Van der Wulp NY, Hoving C, de Vries H. Partner's Influences and Other Correlates of Prenatal Alcohol Use. Matern Child Health J. 2015;19(4) :908-16.

54. Mullally A, Cleary BJ, Barry J, Fahey TP, Murphy DJ. Prevalence, predictors and perinatal outcomes of peri-conceptional alcohol exposure - retrospective cohort study in an urban obstetric population in Ireland. BMC Pregnancy Childbirth. 2011; 11: 27.

55. Ní Shúilleabhain A, Barry J, Kelly A, O’Kelly F, Darker C, O’Dowd T. Alcohol consumption in pregnancy: Results from the general practice setting. Ir J Med Sci. 2014; 183(2): 231-40.

56. Reynolds CME, Egan B, O’Malley EG, McMahon L, Sheehan SR, Turner MJ. Fetal growth and maternal alcohol consumption during early pregnancy. Eur J Obstet Gynecol Reprod Biol. 2019; 236: 148-53.

57. Dale MTG, Bakketeig LS, Magnus P. Alcohol consumption among first-time mothers and the risk of preterm birth: A cohort study. Ann Epidemiol. 2016; 26(4): 275-82.

58. Mellingen S, Torsheim T, Thuen F. Changes in alcohol use and relationship satisfaction in Norwegian couples during pregnancy. Subst Abus Treat Prev Policy. 2013; 8: 5.

59. Stene-Larsen K, Torgersen L, Strandberg-Larsen K, Normann PT, Vollrath ME. Impact of maternal negative affectivity on light alcohol use and binge drinking during pregnancy. Acta Obstet Gynecol Scand. 2013; 92(12): 1388-94.

60. Zuccolo L, DeRoo LA, Wills AK, Smith GD, Suren P, Roth C, et al. Pre-conception and prenatal alcohol exposure from mothers and fathers drinking and head circumference: Results from the Norwegian Mother-Child Study (MoBa). Sci Rep. 2016; 7: 39535.

61. Esper LH, Furtado EF. Stressful life events and alcohol consumption in pregnant women: A crosssectional survey. Midwifery. 2019;71: 27-32.

62. Hackbarth BB, Ferreira JA, Carstens HP, Amaral AR, Silva MR, Silva JC, et al. Suscetibilidade à prematuridade: Investigação de fatores comportamentais, genéticos, médicos e sociodemográficos. Rev Bras Ginecol e Obstet. 2015; 37(8): 353-8.

63. Sbrana M, Grandi C, Brazan M, Junquera N, Nascimento MS, Barbieri MA, et al. Consumo de álcool durante a gravidez e resultados perinatais: Um estudo de coorte. Sao Paulo Med J. 2016; 134(2): 14652.

64. Veloso LUP, Monteiro CF de S. Prevalence and factors associated with alcohol use among pregnant adolescents. Rev Lat Am Enfermagem. 2013; 21(1): 433-41.

65. Doherty E, Wiggers J, Wolfenden L, Anderson AE, Crooks K, Tsang TW, et al. Antenatal care for alcohol consumption during pregnancy: Pregnant women's reported receipt of care and associated characteristics. BMC Pregnancy Childbirth. 2019; 19(1): 299.

66. Dumas A, Toutain S, Simmat-Durand L. Alcohol Use during Pregnancy or Breastfeeding: A National Survey in France. J Women's Heal. 2017; 26(7): 798-805.

67. González-Mesa E, Blasco-Alonso M, Gálvez Montes M, Lozano Bravo I, Merino-Galdón F, CuencaCampos F, et al. High levels of alcohol consumption in pregnant women from a touristic area of Southern Spain. J Obstet Gynaecol (Lahore). 2015; 35(8): 821-4.

68. Louw JG, Tomlinson M, Olivier L. Unrealistic optimism with regard to drinking during pregnancy among women of childbearing age in a South African community. South African J Psychol. 2018; 48(2): 219-29.

69. Mpelo M, Kibusi SM, Moshi F, Nyundo A, Ntwenya JE, Mpondo BCT. Prevalence and factors influencing alcohol use in pregnancy among women attending antenatal care in Dodoma region, Tanzania: A cross-sectional study. J Pregnancy. 2018; 2018: 8580318.

70. Parackal S, Parackal M, Harraway J. Associated factors of drinking prior to recognising pregnancy and risky drinking among New Zealand women aged 18 to 35 years. Int J Environ Res Public Health. 2019; 16(10): 1822.

71. Symon A, Rankin J, Butcher G, Smith L, Cochrane L. Evaluation of a retrospective diary for periconceptual and mid-pregnancy drinking in Scotland: a cross-sectional study. Acta Obstet Gynecol Scand. 2017; 96(1):53-60.

72. Bazzo S, Battistella G, Riscica P, Moino G, Pozzo GD, Bottarel M, et al. Reliability of a self-report Italian version of the AUDIT-C questionnaire, used to estimate alcohol consumption by pregnant women in an obstetric setting Valutazione dell'affidabilità della versione italiana del questionario AUDIT-C per la rilevazione del consumo di alcol in gravidanza. Riv Psichiatr 2015; 50(2): 89-94.

73. de Vries MM, Joubert B, Cloete M, Roux S, Baca BA, Hasken JM, et al. Indicated prevention of fetal 
alcohol spectrum disorders in South Africa: Effectiveness of case management. Int J Environ Res Public Health. 2015;13(1): ijerph13010076.

74. Williams AD, Nkombo Y, Nkodia G, Leonardson G, Burd L. Prenatal alcohol exposure in the Republic of the Congo: Prevalence and screening strategies. Birth Defects Res Part A - Clin Mol Teratol. 2013; 97(7): 489-96.

75. Hutson JR, Magri R, Gareri JN, Koren G. The Incidence of Prenatal Alcohol Exposure in Montevideo Uruguay As Determined by Meconium Analysis. 2010; 32(3): 311-7.

76. Assanangkornchai S, Saingam D, Apakupakul N, Edwards JG. Alcohol consumption, smoking, and drug use in pregnancy: Prevalence and risk factors in Southern Thailand. Asia-Pacific Psychiatry. 2017; 9(1).

77. Azurmendi-Funes ML, Martínez-Villanueva M, Delgado-Marín JL, Ramis R, Sánchez-Sauco MF, López-Hernández FA, et al. An Integrative Screening Tool of Alcohol Exposure during Early Pregnancy: Combining of the CDT Biomarker with Green Page Questionnaire. Alcohol Alcohol. 2019; 54(6): 599-608.

78. Cluver CA, Charles W, van der Merwe C, Bezuidenhout H, Nel D, Groenewald C, et al. The association of prenatal alcohol exposure on the cognitive abilities and behaviour profiles of 4-year-old children: a prospective cohort study. BJOG An Int J Obstet Gynaecol. 2019; 126(13): 1588-97.

79. Chambers CD, Yevtushok L, Zymak-Zakutnya N, Korzhynskyy Y, Ostapchuk L, Akhmedzhanova D, et al. Prevalence and Predictors of Maternal Alcohol Consumption in Two Regions of Ukraine Alcohol Clin Exp Res. 2014; 38(4): 1012-1019

80. Kim O, Park K. Prenatal alcohol consumption and knowledge about alcohol consumption and fetal alcohol syndrome in Korean women. Nurs Heal Sci. 2011; 13(3): 303-8.

81. López MB, Lichtenberger A, Conde K, Cremonte M. Propriedades psicométricas de instrumentos de triagem de consumo de álcool durante gestação na Argentina. Rev Bras Ginecol e Obstet. 2017; 39(7): 322-9.

82. Seib CA, Daglish M, Heath R, Booker C, Reid C, Fraser J. Screening for alcohol and drug use in pregnancy. Midwifery. 2012; 28(6): 760-4.

83. Sarkar M, Einarson T, Koren G. Comparing the effectiveness of TWEAK and T-ACE in determining problem drinkers in pregnancy. Alcohol Alcohol. 2010; 45(4): 356-60.

84. Vythilingum B, Roos A, Faure SC, Geerts L, Stein DJ. Risk factors for substance use in pregnant women in South Africa. S Afr Med J. 2012; 102(11 Pt 1): 851-4.

85. Iversen ML, Sørensen NO, Broberg L, Damm P, Hedegaard M, Tabor A, et al. Alcohol consumption and binge drinking in early pregnancy. A cross-sectional study with data from the Copenhagen Pregnancy Cohort. BMC Pregnancy Childbirth. 2015;15: 327.

86. Dimitriu M, Ionescu CA, Matei A, Viezuina R, Rosu G, Ilinca C, et al. The problems associated with adolescent pregnancy in Romania: A cross-sectional study. J Eval Clin Pract. 2019; 25(1): 117-24.

87. Schoeps A, Peterson ER, Mia Y, Waldie KE, Underwood L, D'Souza S, et al. Prenatal alcohol consumption and infant and child behavior: Evidence from the Growing Up in New Zealand Cohort. Early Hum Dev. 2018; 123: 22-9.

88. Alshaarawy O, Breslau N, Anthony JC. Monthly Estimates of Alcohol Drinking During Pregnancy: United States, 2002-2011. Stud. Alcohol Drugs. 2016; 77(2): 272-6.

89. May PA, Blankenship J, Marais AS, Gossage JP, Kalberg WO, Joubert B, et al. Maternal alcohol consumption producing fetal alcohol spectrum disorders (FASD): Quantity, frequency, and timing of drinking. Drug Alcohol Depend. 2013; 133(2): 502-12.

90. Eaton LA, Pitpitan E V., Kalichman SC, Sikkema KJ, Skinner D, Watt MH, et al. Beliefs about fetal alcohol spectrum disorder among men and women at alcohol serving establishments in South Africa. Am J Drug Alcohol Abuse. 2014; 40(2): 87-94.

91. O’Leary CM, Taylor C, Zubrick SR, Kurinczuk JJ, Bower C. Prenatal alcohol exposure and educational achievement in children aged 8-9 years. Pediatrics. 2013; 132(2): e468-75.

92. Burns L, Black E, Powers JR, Loxton D, Elliott E, Shakeshaft A, et al. Geographic and Maternal Characteristics Associated with Alcohol Use in Pregnancy. Alcohol Clin Exp Res. 2011; 35(7): 12307

93. Wilson J, Tay RY, McCormack C, Allsop S, Najman J, Burns L, et al. Alcohol consumption by breastfeeding mothers: Frequency, correlates and infant outcomes. Drug Alcohol Rev. 2017; 36(5): 667-76.

94. Walker MJ, Al-Sahab B, Islam F, Tamim H. The epidemiology of alcohol utilization during pregnancy: An analysis of the Canadian Maternity Experiences Survey (MES). BMC Pregnancy Childbirth. 2011;11: 52.

95. Pei L, Kang Y, Cheng Y, Yan H. The association of maternal lifestyle with birth defects in Shaanxi Province, Northwest China. PLoS One. 2015; 10(9): e0139452. 
96. Petković G, Barišić I. Prevalence of fetal alcohol syndrome and maternal characteristics in a sample of schoolchildren from a rural province of Croatia. Int J Environ Res Public Health. 2013; 10(4): 154761.

97. Sanvisens A, Robert N, Hernández J, Zuluaga P, Farré M, Coroleu W, et al. Alcohol consumption during pregnancy: Analysis of two direct metabolites of ethanol in meconium. Int J Mol Sci. 2016; 17(3): 417.

98. Adusi-Poku Y, Edusei AK, Bonney AA, Tagbor H, Nakua E, Otupiri E. Pregnant Women and Alcohol Use in the Bosomtwe District of the Ashanti Region-Ghana. Afr J Reprod Health. 2012; 16(1): 55-60.

99. Adusi-Poku Y, Bonney AA, Antwi GD. Where, When And What Type Of Alcohol Do Pregnant Women Drink? Ghana Med J. 2013; 47(1): 35-9.

100. Esposito G, Ambrosio R, Napolitano F, Di Giuseppe G. Women's knowledge, attitudes and behavior about maternal risk factors in pregnancy. PLoS One. 2015; 10(12): e0145873.

101. Ikehara S, Kimura T, Kakigano A, Sato T, Iso H, Saito H, et al. Association between maternal alcohol consumption during pregnancy and risk of preterm delivery: the Japan Environment and Children's Study. BJOG An Int J Obstet Gynaecol. 2019; 126(12): 1448-1454.

102. Miyake Y, Tanaka K, Okubo H, Sasaki S, Arakawa M. Alcohol consumption during pregnancy and birth outcomes: The Kyushu Okinawa Maternal and Child Health Study. BMC Pregnancy Childbirth. 2014; 14(1): 79 .

103. Mallard SR, Connor JL, Houghton LA. Maternal factors associated with heavy periconceptional alcohol intake and drinking following pregnancy recognition: A post-partum survey of New Zealand women. Drug Alcohol Rev. 2013; 32(4): 389-97.

104. Panstw Zakl R, Hamułka J, Zielińska MA, Chądzyńska K. The combined effects of alcohol and tobacco use during pregnancy on birth outcomes. Rocz Panstw Zakl Hig. 2018; 69(1): 45-54.

105. Wojtyła A, Kapka-Skrzypczak L, Diatczyk J, Fronczak A, Paprzycki P. Alcohol-related Developmental Origin of Adult Health - population studies in Poland among mothers and newborns (2010-2012). Ann Agric Environ Med. 2012; 19(3): 365-377.

106. Dupraz J, Graff V, Barasche J, Etter JF, Boulvain M. Tobacco and alcohol during pregnancy: Prevalence and determinants in Geneva in 2008. Swiss Med Wkly. 2013;143: w13795.

107. Yen CF, Yang MS, Lai CY, Chen CC, Yeh YC, Wang PW. Alcohol consumption after the recognition of pregnancy and correlated factors among indigenous pregnant women in Taiwan. Matern Child Health J. 2012; 16(2): 528-38.

108. M'soka NC, Mabuza LH, Pretorius D. Cultural and health beliefs of pregnant women in Zambia regarding pregnancy and child birth. Curationis. 2015; 38(1): 1232.

109. Murphy DJ, Dunney C, Mullally A, Adnan N, Deane R. Population-based study of smoking behaviour throughout pregnancy and adverse perinatal outcomes. Int J Environ Res Public Health. 2013;10(9): 3855-67.

110. Marangoni SR, Lúcia M, De Oliveira F. Fatores Desencadeantes Do Uso De Drogas De Abuso Em Mulheres. Texto Context Enferm. 2013; 22(3): 662-70.

111. . Martinelli JL, Germano CMR, de Avó LRDS, Fontanella BJB, Melo DG. Alcohol Consumption During Pregnancy in Brazil: Elements of an Interpretive Approach. Qual Health Res. 2021; 31(11): 2123-2134.

112. Lyall V, Wolfson L, Reid N, Poole N, Moritz KM, Egert S, Browne AJ, Askew DA. "The Problem Is that We Hear a Bit of Everything...": A Qualitative Systematic Review of Factors Associated with Alcohol Use, Reduction, and Abstinence in Pregnancy. Int J Environ Res Public Health. 2021;18(7): 3445 . 


\section{Material Suplementar}

Quadro Suplementar 1 - Características dos questionários validados encontrados nos estudos incluídos

\begin{tabular}{|c|c|c|c|c|}
\hline Questionário & $\begin{array}{l}\text { Objetivo e } \\
\text { Público-alvo } \\
\end{array}$ & Forma de avaliação & Mensuração & $\begin{array}{l}\text { Amostra } \\
\text { validação }\end{array}$ \\
\hline 5Ps, 2003 & $\begin{array}{l}\text { Risco de consumo } \\
\text { de álcool tanto } \\
\text { pela gestante } \\
\text { quanto por pessoas } \\
\text { próximas. }\end{array}$ & $\begin{array}{l}\text { Opções de respostas: } 1 \text { a } \\
3 \text { (sim, não ou "sem } \\
\text { resposta"); } 4 \text { e } 5 \text { (de } \\
\text { modo algum, raramente, } \\
\text { algumas vezes ou } \\
\text { frequentemente). }\end{array}$ & $\begin{array}{l}\text { Qualquer resposta } \\
\text { positiva às } 5 \\
\text { perguntas, } \\
\text { incluindo } \\
\text { "raramente" nas } \\
\text { questões } 4 \text { e } 5 \text {, } \\
\text { seleciona uma } \\
\text { mulher grávida } \\
\text { para uma breve } \\
\text { intervenção. }\end{array}$ & $\begin{array}{l}\text { Mulheres grávidas. } \\
\text { Centros de saúde } \\
\text { comunitários } \\
\text { (n=1979) e clínicas } \\
\text { privadas (n=2449). } \\
\text { MA, EUA. }\end{array}$ \\
\hline ASSIST, 2002* & $\begin{array}{c}\text { Rastreio do } \\
\text { consumo de álcool } \\
\text { e outras } \\
\text { substâncias na } \\
\text { população em } \\
\text { geral. }\end{array}$ & $\begin{array}{l}\text { Contém } 8 \text { questões } \\
\text { sobre o uso de } \\
\text { substâncias psicoativas } \\
\text { (tabaco, álcool, } \\
\text { maconha, estimulantes, } \\
\text { sedativos, inalantes, } \\
\text { alucinógenos e } \\
\text { opiáceos). }\end{array}$ & $\begin{array}{l}\text { Cada resposta } \\
\text { corresponde a um } \\
\text { escore }(0 \text { a } 4) \text {. A } \\
\text { soma dos escores } \\
\text { varia de } 0 \text { a } 20 \text {, em } \\
\text { que de } 0 \text { a } 3: \\
\text { indicativa de uso } \\
\text { ocasional; de } 4 \text { a } \\
\text { 15: indicativa de } \\
\text { Abuso; } \geq 16: \\
\text { sugestiva de } \\
\text { dependência. }\end{array}$ & $\begin{array}{l}\text { Participaram } \\
\text { voluntários de } \\
\text { vários países: } \\
\text { Austrália, Brasil, } \\
\text { Irlanda, Índia, } \\
\text { Israel, Território } \\
\text { Palestino, Porto } \\
\text { Rico, Reino Unido e } \\
\text { Zimbábue (n=236). }\end{array}$ \\
\hline $\begin{array}{c}\text { AUDIT, 1982* } \\
\text { AUDIT-C, 1990* }\end{array}$ & $\begin{array}{c}\text { Rastreio do } \\
\text { consumo de álcool } \\
\text { na população em } \\
\text { geral. }\end{array}$ & $\begin{array}{l}\text { AUDIT: } 10 \text { questões: } 3 \\
\text { sobre quantidade e a } \\
\text { frequência do uso de } \\
\text { álcool; } 3 \text { sobre } \\
\text { dependência; e } 4 \text { sobre } \\
\text { problemas recentes } \\
\text { relacionados ao } \\
\text { consumo do álcool. } \\
\text { AUDIT-C: Versão } \\
\text { resumida. Contém } 3 \\
\text { questões. }\end{array}$ & $\begin{array}{c}\text { AUDIT: cada } \\
\text { questão tem uma } \\
\text { margem de } 0 \text { a } 4 \\
\text { com pontuação } \\
\text { final de } 0 \text { a } 40 \\
\text { pontos. AUDIT-C: } \\
\text { Pontuação de } 0 \text { a } \\
12 \text { pontos. Ambos } \\
\text { classificam em: } \\
\text { baixo risco; risco } \\
\text { moderado; alto } \\
\text { risco; risco severo. }\end{array}$ & $\begin{array}{l}\text { AUDIT: pacientes } \\
\text { de diferentes } \\
\text { serviços médios em } \\
\text { seis países } \\
(\mathrm{n}=2000) \text {. AUDIT- } \\
\text { C: pacientes de três } \\
\text { clínicas de medicina } \\
\text { geral para veteranos } \\
\text { militares dos EUA } \\
(\mathrm{n}=393) .\end{array}$ \\
\hline CAGE, 1974* & $\begin{array}{l}\text { Rastreio do } \\
\text { consumo de álcool } \\
\text { na população } \\
\text { geral. }\end{array}$ & $\begin{array}{l}\text { Contém } 4 \text { questões com } \\
\text { alternativas de respostas } \\
\text { dicotômicas (sim e não). }\end{array}$ & $\begin{array}{l}\text { Pelo menos uma } \\
\text { resposta } \\
\text { afirmativa: } \\
\text { suspeita de } \\
\text { problemas com o } \\
\text { álcool. Duas ou } \\
\text { mais respostas } \\
\text { afirmativas: } \\
\text { problemas com o } \\
\text { álcool. }\end{array}$ & $\begin{array}{c}\text { Pacientes } \\
\text { psiquiátricos de um } \\
\text { hospital para } \\
\text { veteranos militares } \\
\text { que foram admitidos } \\
\text { ao longo de um ano } \\
\text { (n=366). } \\
\text { Durham, N.C., } \\
\text { EUA. }\end{array}$ \\
\hline CRAFFT, 1999 & $\begin{array}{c}\text { Rastreio do } \\
\text { consumo de álcool } \\
\text { e outras } \\
\text { substâncias por } \\
\text { adolescentes. }\end{array}$ & $\begin{array}{l}\text { Contém } 6 \text { questões com } \\
\text { alternativas de respostas } \\
\text { dicotômicas (sim e não). }\end{array}$ & $\begin{array}{c}\text { Cada resposta } \\
\text { afirmativa marca } 1 \\
\text { ponto e é positivo } \\
\text { com uma } \\
\text { pontuação de } 2 \text { ou } \\
\text { mais pontos. } \\
\end{array}$ & $\begin{array}{c}\text { Adolescentes ( } \mathrm{n}=99) \\
\text { que foram atendidos } \\
\text { por consultas de } \\
\text { rotina no Hospital } \\
\text { Infantil de Boston, } \\
\text { Boston, MA, EUA. }\end{array}$ \\
\hline $\begin{array}{l}\text { GREEN PAGE } \\
\text { (GP), } 2012\end{array}$ & $\begin{array}{c}\text { Identifica } \\
\text { exposições } \\
\text { ambientais em } \\
\text { mulheres grávidas, } \\
\text { incluindo álcool. }\end{array}$ & $\begin{array}{l}\text { Permite a conversão do } \\
\text { consumo de álcool de } \\
\text { acordo com os tipos de } \\
\text { bebidas alcoólicas } \\
\text { consumidas e a } \\
\text { frequência em g/dia de } \\
\text { álcool. }\end{array}$ & $\begin{array}{c}\text { Permite a } \\
\text { conversão do } \\
\text { consumo de álcool } \\
\text { em g/dia durante } \\
\text { períodos críticos. }\end{array}$ & $\begin{array}{c}\text { Acompanhou } \\
\text { gestações desde o } 1^{\circ} \\
\text { trimestre até o } \\
\text { nascimento e } \\
\text { avaliou a } \\
\text { circunferência da } \\
\text { cabeça nos neonatos } \\
(\mathrm{n}=419) .\end{array}$ \\
\hline
\end{tabular}


Vittalle - Revista de Ciências da Saúde v. 33, n. 3 (2021) 112-133

\begin{tabular}{|c|c|c|c|c|}
\hline $\begin{array}{l}\text { NIDA Quick } \\
\text { Screen, } 2012\end{array}$ & $\begin{array}{c}\text { Rastreio do } \\
\text { consumo de álcool } \\
\text { e outras } \\
\text { substâncias na } \\
\text { população em } \\
\text { geral. } \\
\end{array}$ & $\begin{array}{c}\text { Pergunta única: } \\
\text { "Quantas vezes no ano } \\
\text { passado você usou uma } \\
\text { droga ilegal ou uma } \\
\text { prescrição médica por } \\
\text { razões não médicas?" }\end{array}$ & $\begin{array}{l}\text { Uma resposta de } \\
\text { pelo menos } 1 \mathrm{vez} \\
\text { foi considerada } \\
\text { positiva para o uso } \\
\text { de drogas. * }\end{array}$ & $\begin{array}{c}\text { Pacientes de clínica } \\
\text { geral }(\mathrm{n}=303) \\
\text { Boston University } \\
\text { Medical Center, } \\
\text { Boston, MA, EUA. } \\
\text { 2006/2007. }\end{array}$ \\
\hline RAPS-QF, 1995 & $\begin{array}{l}\text { Rastreio do } \\
\text { consumo de álcool } \\
\text { na população } \\
\text { geral. }\end{array}$ & $\begin{array}{l}6 \text { itens: remorso, } \\
\text { amnésia, desempenho, } \\
\text { consumo matinal, } \\
\text { quantidade e frequência } \\
\text { de consumo de álcool. }\end{array}$ & $\begin{array}{l}\text { Se pelo menos } \\
\text { uma resposta a } \\
\text { essas perguntas for } \\
\text { afirmativa há } \\
\text { suspeita } \\
\text { de problemas com } \\
\text { o álcool. } \\
\end{array}$ & $\begin{array}{l}\text { Pacientes de pronto } \\
\text { atendimento } \\
(\mathrm{n}=1.330) . \\
\text { Mississippi Medical } \\
\text { Center, MS, EUA. }\end{array}$ \\
\hline SURP-P, 2010 & $\begin{array}{c}\text { Rastreio do } \\
\text { consumo de álcool } \\
\text { e outras } \\
\text { substâncias por } \\
\text { mulheres grávidas. }\end{array}$ & $\begin{array}{c}\text { Contém } 16 \text { perguntas } \\
\text { elaboradas com base em } \\
\text { outros questionários: } \\
\text { Índice de Gravidade da } \\
\text { Dependência, 4Ps Plus e } \\
\text { TWEAK. * }\end{array}$ & $\begin{array}{l}\text { As respostas são } \\
\text { sim ou não, a } \\
\text { menos que } \\
\text { especificado de } \\
\text { outra forma. A } \\
\text { pontuação se dá } \\
\text { conforme o } \\
\text { protocolo em que } \\
\text { cada pergunta foi } \\
\text { baseada. } * \\
\end{array}$ & $\begin{array}{c}\text { Gestantes de três } \\
\text { clínicas de saúde } \\
\text { reprodutiva } \\
\text { (n=1064). Escola de } \\
\text { Medicina de Yale e } \\
\text { Hospital Bridgeport, } \\
\text { CT, EUA. }\end{array}$ \\
\hline T-ACE, 1989* & $\begin{array}{l}\text { Rastreio do } \\
\text { consumo de álcool } \\
\text { por mulheres } \\
\text { grávidas. }\end{array}$ & $\begin{array}{l}\text { Contém } 4 \text { questões } \\
\text { principais intercaladas } \\
\text { com perguntas extras } \\
\text { sobre a gestação. }\end{array}$ & $\begin{array}{l}\text { A pontuação varia } \\
\text { de } 0 \text { a } 5 \text { pontos. } \\
\text { Pontuação total } \geq \\
2 \text { caracteriza a mãe } \\
\text { como T-ACE } \\
\text { positiva. * }\end{array}$ & $\begin{array}{l}\text { Gestantes ( } \mathrm{n}=1065 . \\
\text { Entrevistas: clínica } \\
\text { obstétrica em } \\
\text { Detroit, MI, EUA. }\end{array}$ \\
\hline TLFB, 1992 & $\begin{array}{l}\text { Avalia o consumo } \\
\text { de álcool com } \\
\text { estimativas diárias. } \\
\text { Usado na } \\
\text { população em } \\
\text { geral. }\end{array}$ & $\begin{array}{l}\text { Rastreia estimativas } \\
\text { retrospectivas do } \\
\text { consumo diário durante } \\
\text { um período } \\
\text { especificado, que pode } \\
\text { variar até } 12 \text { meses a } \\
\text { partir da data da } \\
\text { entrevista. }\end{array}$ & $\begin{array}{c}\text { É possível captar } \\
\text { informações sobre } \\
\text { padrão, } \\
\text { variabilidade e } \\
\text { magnitude do } \\
\text { consumo. * }\end{array}$ & $\begin{array}{c}\text { Bebedores normais } \\
(\mathrm{n}=62), \text { estudantes } \\
(\mathrm{n}=80 \text { e } \mathrm{n}=48), \\
\text { alcoolistas } \\
\text { residenciais }(\mathrm{n}=12), \\
\text { ambulatoriais } \\
(\mathrm{n}=12), \text { internados } \\
(\mathrm{n}=12) ; \text { veteranos } \\
\text { militares }(\mathrm{n}=26) .\end{array}$ \\
\hline TWEAK, 1979 & $\begin{array}{l}\text { Rastreio do } \\
\text { consumo de álcool } \\
\text { por mulheres } \\
\text { grávidas. }\end{array}$ & $\begin{array}{l}\text { Contém } 5 \text { questões } \\
\text { principais intercaladas } \\
\text { com perguntas extras } \\
\text { sobre a gestação. }\end{array}$ & $\begin{array}{c}\text { A pontuação total } \\
\text { pode variar de } 0 \text { a } \\
7 \text { pontos. } \\
\text { Pontuação total } \geq \\
2 \text { caracteriza uma } \\
\text { bebedora de risco. }\end{array}$ & $\begin{array}{c}\text { Mulheres atendidas } \\
\text { em uma clínica } \\
\text { obstétrica- } \\
\text { ginecológica } \\
(\mathrm{n}=499) \text {. Buffalo, } \\
\text { NY, EUA. }\end{array}$ \\
\hline WIDUS, 2012 & $\begin{array}{c}\text { Rastreio do } \\
\text { consumo de álcool } \\
\text { e outras } \\
\text { substâncias por } \\
\text { mulheres grávidas. }\end{array}$ & $\begin{array}{c}\text { Questionário de } 6 \text { itens } \\
\text { que foram validados a } \\
\text { partir de uma lista } \\
\text { inicial contendo } 74 \\
\text { itens. } *\end{array}$ & $\begin{array}{c}\text { Todos os itens } \\
\text { usam um padrão } \\
\text { de resposta de } \\
\text { verdadeiro ou } \\
\text { falso para rastrear } \\
\text { o uso de álcool e } \\
\text { outras drogas nos } \\
3 \text { meses finais da } \\
\text { gestação. } * \\
\end{array}$ & $\begin{array}{l}\text { Mulheres afro- } \\
\text { americanas de baixa } \\
\text { renda }(\mathrm{n}=400) \\
\text { recrutadas em um } \\
\text { hospital obstétrico. } \\
\text { Detroit, MI, EUA. }\end{array}$ \\
\hline Q-F, 2005 & $\begin{array}{c}\text { Estimativa do } \\
\text { consumo de álcool. }\end{array}$ & $\begin{array}{l}2 \text { perguntas: quantas } \\
\text { doses padrão } \\
\text { geralmente são } \\
\text { consumidas? Com que } \\
\text { frequência eles bebem } \\
\text { acima de um limite de } \\
\text { risco? }\end{array}$ & $\begin{array}{l}\text { Permite realizar } \\
\text { uma estimativa do } \\
\text { consumo de } \\
\text { bebidas alcoólicas. }\end{array}$ & $\begin{array}{c}\text { Estudantes } \\
\text { universitários } \\
\text { (n=15.797) de } 27 \\
\text { universidades dos } \\
\text { EUA. Coleta entre } \\
\text { os anos de } 1949 \text { e } \\
1951 . \\
\text { Subclassificações } \\
\text { por sexo, religião, } \\
\text { renda familiar. Uso } \\
\text { de escalas de } \\
\text { consumo habitual de } \\
\text { álcool e } \\
\text { complicações } \\
\end{array}$ \\
\hline
\end{tabular}


M. V. S. Amorim et al. / Vittalle v. 33 n. 3 (2021) 112-133

\begin{tabular}{|c|c|c|c|c|}
\hline & & & & $\begin{array}{l}\text { sociais. Centro de } \\
\text { Estudos de Álcool } \\
\text { de Yale. }\end{array}$ \\
\hline SAQ, 1975 & $\begin{array}{c}\text { Rastreio do } \\
\text { conhecimento e } \\
\text { consumo de álcool } \\
\text { entre alunos. }\end{array}$ & $\begin{array}{l}\text { Questionário para ser } \\
\text { aplicado na forma de } \\
\text { autorrelato, contém } 70 \\
\text { questões divididas em } \\
\text { quatro seções: } \\
\text { quantidade/frequência; } \\
\text { problemas resultantes } \\
\text { de beber; conhecimento } \\
\text { acerca do álcool e } \\
\text { comportamento } \\
\text { relacionado ao consumo } \\
\text { de álcool. }\end{array}$ & $\begin{array}{c}\text { Para os itens } \\
\text { relacionados ao } \\
\text { conhecimento } \\
\text { acerca do álcool } \\
\text { utiliza respostas de } \\
\text { verdadeiro ou } \\
\text { falso. Os demais } \\
\text { itens coletam } \\
\text { informações sobre } \\
\text { padrão, } \\
\text { variabilidade e } \\
\text { magnitude do } \\
\text { consumo. * }\end{array}$ & $\begin{array}{c}\text { Alunos e } \\
\text { funcionários } \\
(\mathrm{n}=1.128) \text { de } 13 \\
\text { faculdades dos EUA } \\
\text { foram escolhidos } \\
\text { para representar a } \\
\text { amostra. }\end{array}$ \\
\hline
\end{tabular}

Nota: * Possui validação no Brasil, para maiores informações consultar referência original disponível na Tabela 2 do material suplementar

Legenda: 4Ps, 4 questões sobre Parents, Partner, Past, Present (pais, parceiro, passado e presente). 5Ps, 5 questões sobre Parents, Partner, Past, Presen, Peerst (pais, parceiro, passado, presente e pares). ASSIST, Alcohol, Smoking and Substance Involvement Screening Test (teste para rastrear o envolvimento com álcool, fumo e substâncias). AUDIT, Alcohol Use Disorders Identification Test (teste para identificação de problemas relacionados ao uso de álcool). CAGE, Cut-down; Annoyed; Guilty; Eye-opener (redução; aborrecimento; culpado; ingesta matinal, para "abrir o olho"). CRAFFT, Car; Relax; Alone; Forget; Friends; Trouble (seis palavras chaves contidas nas perguntas: carro, relaxar, sozinho, esquecer, amigos, problema). EUA, Estados Unidos da América. g/dia: gramas por dia. NIDA, National Institute on Drug Abuse.Q-F, Standard quantity-frequency. RAPS-QF, Rapid Alcohol Problems Screen - Quantity Frequency (teste rápido de problemas com álcool - frequência e quantidade). SAQ, The Student Alcohol Questionnaire. SURP-P, Substance Use Risk Profile-Pregnancy (perfil de risco de uso de substâncias gravidez).T-ACE, Tolerance; Annoyed; Cut-down; Eye-opener (tolerância; aborrecimento; redução; ingesta matinal, para "abrir o olho", relacionada à persistência e dependência).TLFB, Timeline Followback. TWEAK, Tolerance; Worry; Eye-opener; Annoyed; Cut-down (tolerância, preocupação, ingesta matinal, para "abrir o olho", aborrecimento, redução). WIDUS, Wayne Indirect Drug Use Screener (rastreador indireto de uso de drogas da Wayne). 
Quadro Suplementar 2 - Referências completas dos questionários encontrados

\begin{tabular}{|c|c|}
\hline ste & \\
\hline 5Ps & $\begin{array}{l}\text { Original: Kennedy, C. et al. Improving Screening for Alcohol Use During Pregnancy: The } \\
\text { Massachusetts ASAP Program. Maternal and Child Health Journal, v. 8(3), p. 137-147, } 2004 . \\
\text { doi:10.1023/b:maci.0000037647.78420.e3 }\end{array}$ \\
\hline ASSIST & $\begin{array}{l}\text { Original: Group, W. A. W. The Alcohol, Smoking and Substance Involvement Screening Test } \\
\text { (ASSIST): development, reliability and feasibility. Addiction, 97(9), 1183-1194., } 2002 . \\
\text { doi:10.1046/j.1360-0443.2002.00185.x. Disponível em: https://www.who.int/management-of- } \\
\text { substance-use/assist. Acesso em: } 14 \text { abr } 2020 . \\
\text { Validação no Brasil: } \\
\text { a) Henrique, I.F.S. et al. Validação da versão brasileira do teste de triagem do envolvimento com álcool } \\
\text { e outras substâncias (ASSIST). Rev. Assoc. Med. Bras., São Paulo, v. 50(2), p.199-206, dez. } 2004 . \\
\text { b) Carminatti, V.P. Validação concorrente e confiabilidade da versão brasileira do ASSIST-WHO } \\
\text { (Alcohol Smoking and Substance } \\
\text { Involvement Screening Test) para adolescentes. 2010. } 107 \text { p. Dissertação (Mestrado em } \\
\text { Psicobiologia) - Universidade Federal de São Paulo (UNIFESP), São Paulo, 2010. }\end{array}$ \\
\hline $\begin{array}{l}\text { AUDIT } \\
\text { AUDIT- } \\
\text { C }\end{array}$ & 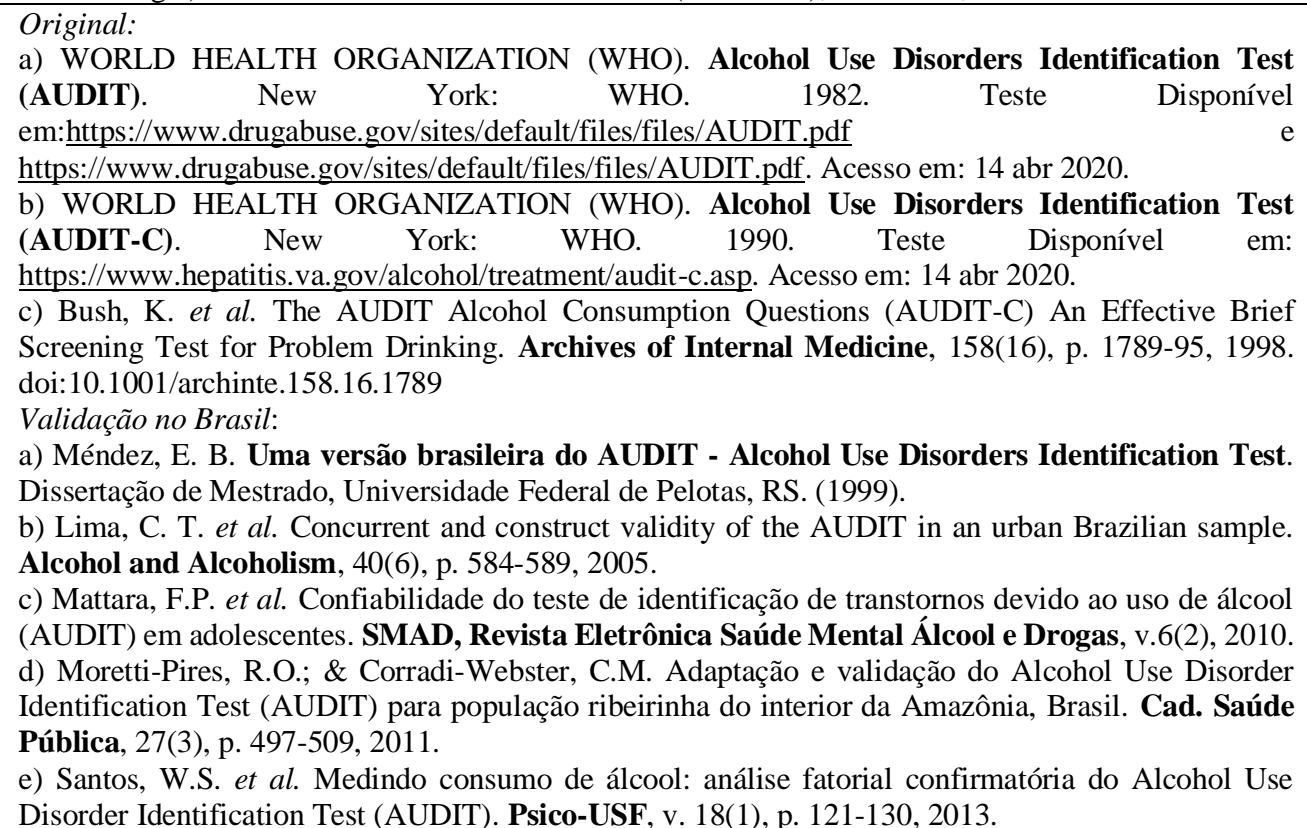 \\
\hline CAGE & $\begin{array}{l}\text { Original: Mayfield, D.; McLeod, G.; \& Hall, P. The CAGE questionnaire: validation of a new } \\
\text { alcoholism screening instrument. The American Journal of Psychiatry, 131(10), 1121-1123, } 1974 . \\
\text { Validação no Brasil: Masur, J., \& Monteiro, M. Validation of the CAGE alcoholism screening test in } \\
\text { Brazilian psychiatry inpatient hospital setting. J Biol Res; } 16: 215-8,1983 \text {. }\end{array}$ \\
\hline CRAFFT & $\begin{array}{l}\text { Original: a) Knight, J.R. et al. A new brief screen for adolescent substance abuse. Arch. Pediatr. } \\
\text { Adolesc. Med., v. 153, p. 591-596, } 1999 \text {. } \\
\text { b) Knight, J.R. et al. Validity of the CRAFFT substance abuse screening test among adolescent clinic } \\
\text { patients. Arch Pediatr Adolesc Med.; v. 156, p. 607-614, } 2002 \text {. } \\
\text { c) Chang, G. et al. Self-reported alcohol and drug use in pregnant young women: a pilot study of } \\
\text { associated factors and identification. J. Addict. Med., V. 5, p. 221-226, } 2011 \text {. }\end{array}$ \\
\hline $\begin{array}{l}\text { GREEN } \\
\text { PAGE } \\
(\text { GP) }\end{array}$ & $\begin{array}{l}\text { Original: Ortega-García, J.A. et al. Head circumference at birth and exposure to tobacco, alcohol and } \\
\text { illegal drugs during early pregnancy. Childs Nerv Syst., v. 28, p. 433-9, 2012. Teste Disponível em: } \\
\text { http://pehsu.org/wp/wp-content/uploads/MANUAL-DE-HOJA-VERDE.pdf . Acesso em: } 15 \text { abr } 2020 .\end{array}$ \\
\hline NIDA & Original: a) Smith, P. C. et al. A Single-Question Screening Test for Drug Use in Primary Care. \\
\hline Quick & \\
\hline Screen & $\begin{array}{l}\text { b) National Institute on Drug Abuse, 2012. National Institutes of Health, US Department of Health and } \\
\text { Human Services. Resource Guide: Screening for Drug Use in General Medical Settings. National } \\
\text { Institute on Drug Abuse website. Disponível em: https://www.drugabuse.gov/publications/resource- } \\
\text { guide/preface. Acesso em: } 14 \text { abr } 2020 \text {. }\end{array}$ \\
\hline $\begin{array}{l}\text { RAPS- } \\
\text { QF }\end{array}$ & $\begin{array}{l}\text { Original: Cherpitel CJ. Screening for alcohol problems in the emergency room: a rapid alcohol } \\
\text { problems screen. Drug Alcohol Depend. V.40(02), p. 133-137, } 1995 .\end{array}$ \\
\hline SURP-P & $\begin{array}{l}\text { Original: Yonkers, K.A. et al. Screening for prenatal substance use: development of the Substance Use } \\
\text { Risk Profile-Pregnancy scale. Obstet. Gynecol., v. 116, p. } 827-833,2010 .\end{array}$ \\
\hline T-ACE & $\begin{array}{l}\text { Original: Sokol, R. J.; Martier, S. S.; \& Ager, J. W. The T-ACE questions: Practical prenatal detection } \\
\text { of risk-drinking. American Journal of Obstetrics and Gynecology, 160(4), p. 863-870, } 1989 . \\
\text { doi:10.1016/0002-9378(89)90302-5 } \\
\text { Validação no Brasil: a) Fabbri, C.E. Desenvolvimento e validação de instrumento para }\end{array}$ \\
\hline
\end{tabular}




\begin{tabular}{|c|c|}
\hline & $\begin{array}{l}\text { rastreamento do uso nocivo de álcool durante a gravidez (T-Ace). (Dissertação). Ribeirão Preto: } \\
\text { Universidade de São Paulo (USP); } 2002 . \\
\text { b) Fabbri, C.E.; Furtado, E.F.; \& Laprega, M.R. Consumo de álcool na gestação: desempenho da versão } \\
\text { brasileira do questionário T-ACE. Rev Saúde Pública; 41(6), p. 979-84, } 2007 .\end{array}$ \\
\hline TLFB & $\begin{array}{l}\text { Original: Sobell, L.C.; \& Sobell, M.B. Timeline Followback: A technique for assessing self-reported } \\
\text { ethanol consumption. In: Allen, J.; Litten, RZ., editors. Measuring Alcohol Consumption: } \\
\text { Psychosocial and Biological Methods. Totowa, NJ: Humana Press; } 1992 . \text { p. } 41-72 \text {. Guia Disponível } \\
\text { em: https://pubs.niaaa.nih.gov/publications/assessingalcohol/instrumentpdfs/13 tlfb.pdf. Acesso em: } \\
15 \text { abr } 2020 \text {. } \\
\text { Versão modificada para capturar mais informações sobre as bebidas consumidas: } \\
\text { Dukes, K. et al. A modified timeline followback assessment to capture alcohol exposure in pregnant } \\
\text { women: application in the safe passage study. Alcohol; v. } 62 \text {, p. } 17-27,2017 \text {. }\end{array}$ \\
\hline TWEAK & 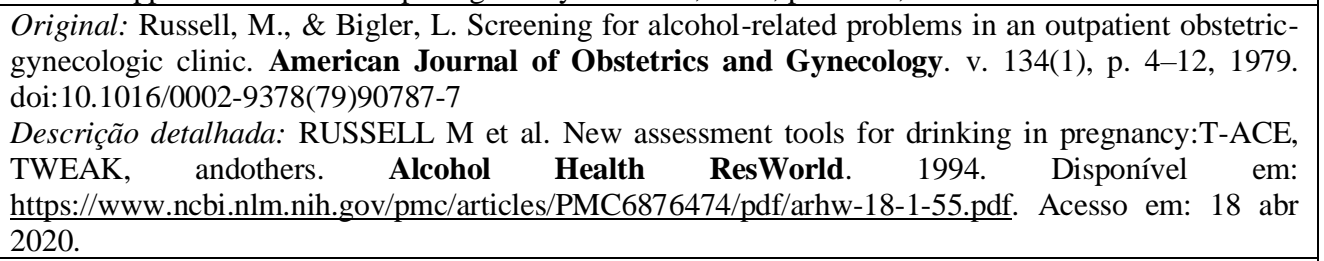 \\
\hline WIDUS & $\begin{array}{l}\text { Original: Ondersma, S. J. et al. Development and preliminary validation of an indirect screener for drug } \\
\text { use in the perinatal period. Addiction, 107(12), p. 2099-2106, 2012. doi:10.1111/j.1360- } \\
0443.2012 .03982 . \mathrm{x}\end{array}$ \\
\hline Q-F & 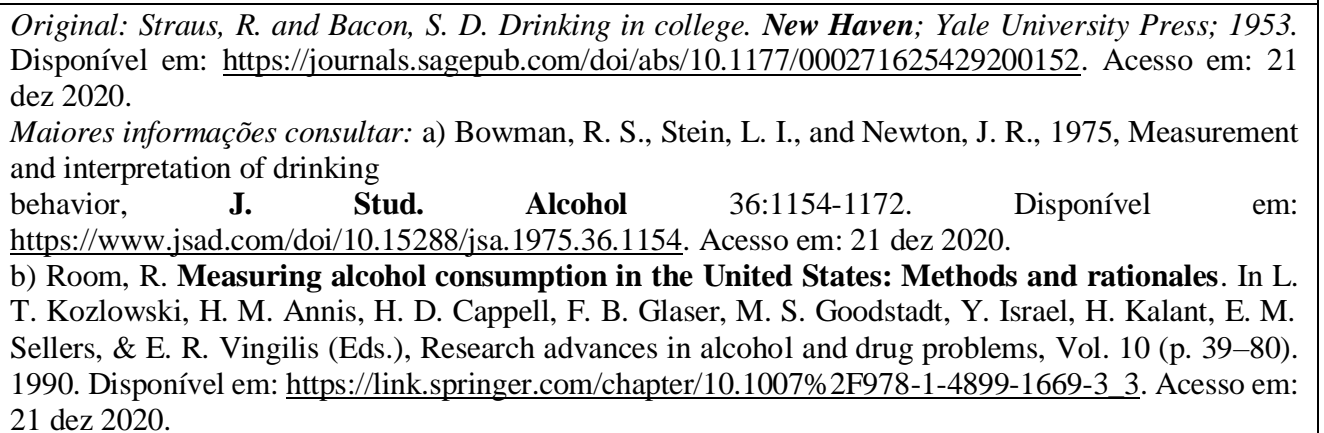 \\
\hline SAQ & $\begin{array}{l}\text { Original: a) Engs, R. C. The Student Alcohol Questionnaire. Bloomington, IN: Department of Health } \\
\text { and Safetv Education. Indiana Univer. } 1975 \text {. } \\
\text { b) Engs, Ruth C. Alcohol knowledge and drinking behavior at thirteen colleges and universities } \\
\text { (And Development of the Student Alcohol Questionnaire). Paper Presented: American College } \\
\text { Health Association National Conference, Philadelphia, Pennsylvania, March 1977. Disponível em: } \\
\text { http://hdl.handle.net/2022/17181. Acesso em: } 21 \text { dez 2020. } \\
\text { Validação no Reino Unido: R.C. Engs. The Student Alcohol Questionnaire (SAQ). Indiana University, } \\
\text { 2002. Disponível em: http://www.indiana.edu/_engs/quet/saq.html. Acesso em: } 21 \text { dez } 2020 .\end{array}$ \\
\hline \multicolumn{2}{|c|}{$\begin{array}{l}\text { Legenda: 5Ps, } 5 \text { questões sobre Parents, Partner, Past, Presen, Peerst. ASSIST, Alcohol, Smoking and } \\
\text { Substance Involvement Screening Test.AUDIT, Alcohol Use Disorders Identification Test. CAGE, Cut- } \\
\text { down; Annoyed; Guilty; Eye-opener.CRAFFT, Car; Relax; Alone; Forget; Friends; Trouble. NIDA, } \\
\text { National Institute on Drug Abuse.Q-F, Standard quantity-frequency RAPS-QF, Rapid Alcohol Problems } \\
\text { Screen - Quantity Frequency. SAQ, The Student Alcohol Questionnaire. SURP-P, Substance Use Risk } \\
\text { Profile-Pregnancy.T-ACE, Tolerance; Annoyed; Cut-down; Eye-opener. TLFB, Timeline Followback. } \\
\text { TWEAK: Tolerance; Worry; Eye-opener; Annoyed; Cut-down. WIDUS, Wayne Indirect Drug Use } \\
\text { Screener }\end{array}$} \\
\hline
\end{tabular}

